Portland State University

PDXScholar

Anthropology Faculty Publications and

Presentations

Anthropology

Summer 2018

\title{
The Galleon Cargo: Accounts in the Colonial Archives
}

\author{
Cameron La Follette \\ Oregon Coast Alliance \\ Douglas Deur \\ Portland State University, deur@pdx.edu \\ Esther González
}

Follow this and additional works at: https://pdxscholar.library.pdx.edu/anth_fac

Part of the Archaeological Anthropology Commons, and the Biological and Physical Anthropology Commons

Let us know how access to this document benefits you.

\section{Citation Details}

Cameron La Follette, Douglas Deur, \& Esther González. (2018). The Galleon Cargo: Accounts in the Colonial Archives. Oregon Historical Quarterly, 119(2), 250-281.

This Article is brought to you for free and open access. It has been accepted for inclusion in Anthropology Faculty Publications and Presentations by an authorized administrator of PDXScholar. Please contact us if we can make this document more accessible: pdxscholar@pdx.edu. 


\title{
The Galleon Cargo
}

\author{
Accounts in the Colonial Archives
}

\section{CAMERON LA FOLLETTE AND DOUGLAS DEUR, WITH ARCHIVAL RESEARCHER ESTHER GONZÁLEZ}

MUCH OF THE FLOTSAM that has often appeared on the beaches of the northern Oregon coast over the past three centuries clearly matches what is documented of average Manila galleon cargos. Native people and, later, Euro-Americans commonly found beeswax chunks large and small; less commonly, they found Chinese blue-and-white porcelain fragments. Both items were mainstays of the Manila trade, signature galleon cargos from the inception of that trade in the mid sixteenth century until its decline roughly two and a half centuries later. Investigations by teams of archaeologists, summarized elsewhere in this special issue, suggest that the debris was most likely left by the wreck of the Santo Cristo de Burgos. 'Additional archival research allows us to compare the cargo carried on that ship with the materials that are known to have been scattered by the Oregon wreck.

A lively beeswax trade existed well before the Spanish entered the Pacific. This trade had relied significantly on Indigenous harvesters in the Philippines, who gathered wax from the nests of the tropical giant honey bee (Apis dorsata) and others. Chinese vessels regularly visited the Philippine coastline to trade for beeswax, centuries before explorers Miguel López de Legázpi and Andrés de Urdaneta interjected Spanish commercial interests into the western Pacific trade during the late sixteenth century. Beeswax being the required base for candles in Catholic liturgy, as well as coveted for household candles, Spanish traders from Urdaneta's time forward carried it as a major cargo on their voyages from Asia to the Americas. ${ }^{2}$ Giovanni Francesco Gemelli Careri, the intrepid Italian voyager who took passage to Acapulco on the Manila galleon of 1697, described the government's removal of unauthorized bales just before sailing, to limit smuggling on his voyage. Careri specifically mentioned that the official, Thomas de Andaya, on one day "caus'd abundance of bales, and parcels of wax to be unshipp'd," with more illegal parcels of wax and packages of goods unloaded the following 


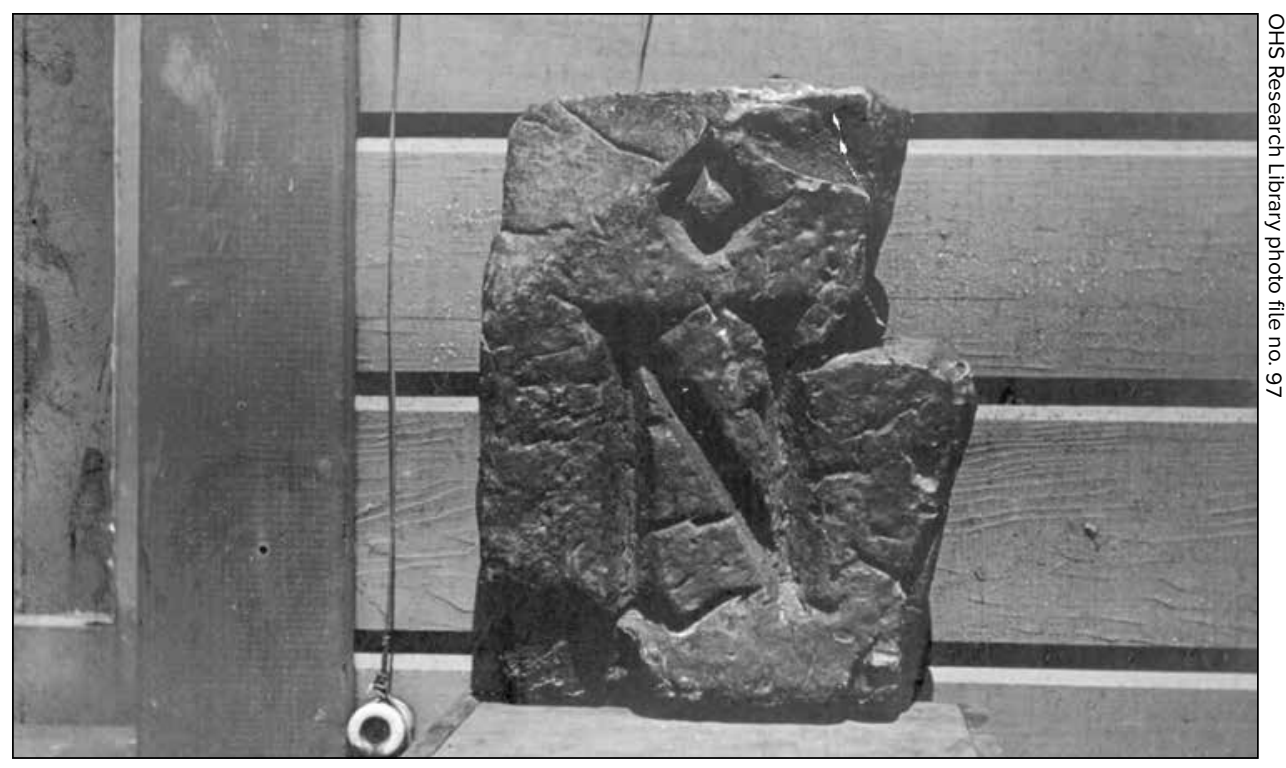

THIS BEESWAX BLOCK was recovered at Nehalem Bay with its shipper's mark still visible. Galleons carried the goods of many colonial merchants and traders in their holds, so these distinctive marks, placed on beeswax and on tightly wrapped bundles of other merchandise, served to distinguish between their cargoes.

day. ${ }^{3}$ Archival documents confirm that other Manila galleons in the same time period often carried substantial cargos of beeswax. ${ }^{4}$ Quantities of beeswax blocks were clearly a common cargo of the Manila trade.

Similarly, the Spanish had a voracious commercial interest in Chinese porcelain, a product coveted throughout Europe and its colonial possessions in the sixteenth, seventeenth, and eighteenth centuries. After some initial experimentation, Manila galleons began carrying large quantities of Chinese porcelain by the end of the sixteenth century. ${ }^{5}$ Mexican entrepreneurs discovered these items were popular and would easily sell in New Spain and beyond, and they communicated this to Philippine merchants, who began to regularly purchase porcelains from Chinese sellers for the Manila trade. From the late sixteenth century through the eighteenth century, exotic Asian porcelains became signifiers of social status throughout New Spain. They were purchased even by communities in remote regions of the Spanish Empire, a range of porcelain types and affordability reflecting a range of income levels. Blue-on-white painted designs became the most popular on tin-glazed, low-fired ceramics (such as mayólica), and they were eventually copied by Mexican artisans as well as imported from 
Manila. ${ }^{6}$ No doubt, any Spanish galleon wrecked on its voyage from Asia would leave an impressive quantity of beeswax and porcelain in its wake.

The large quantities of beeswax and porcelain fragments found on the Oregon coast made a fully loaded Manila galleon the most likely candidate for the "Beeswax Wreck" of Oregon. That fact has long been well established. Before the current archaeological and archival research described in this special issue, speculation and partial consensus in Oregon and in scholarly surveys and studies centered on the San Francisco Xavier of 1705, captained by Don Santiago de Zabalburu, as the ship lost on the Oregon coast. Recent archaeological investigations have upended that interpretation. If that conclusion is verified, the San Francisco has yet to be located, its cargo only described in secondary sources. ${ }^{7}$

Despite of a growing consensus on the identity of the galleon, a complete analysis requires a discussion of the cargoes of both missing ships. Thus, we begin here by surveying the archival documentation of the San Francisco Xavier's cargo. This provides important descriptions of the nature and extent of smuggling - common to the entire Manila galleon trade - and offers a complete 1701 cargo manifest, including shipping marks, which makes it possible to compare with those known from the Nehalem wreck. Studying the San Francisco Xavier's known history also allowed us to review a cargo manifest very similar in time and contents to that of the Santo Cristo de Burgos, for which we found no complete cargo manifest. Still, the archival record also provides tangible clues to that ship's cargo. A reasonable, although not comprehensive, picture of the Santo Cristo's 1693 cargo can be pieced together from references to the San Francisco's cargo and from fragmented archival information about the merchandise the Santo Cristo de Burgos was carrying on its final voyage. Together, the available archival record provides a compelling picture of the cargoes aboard the galleons traveling the Pacific at the time as well as tantalizing facts regarding the one ship that most likely wrecked on the Oregon coast. Cross-checked with the archaeological record, these archival references to specific material items found aboard the two missing galleons are sure to be keystone elements in future shipwreck research.

\section{SAN FRANCISCO XAVIER OF 1705: HISTORY, SMUGGLING, AND CARGO}

Much of the archival documentation of the San Francisco Xavier's history showcases the unrelenting struggles and ingenious subterfuges used to increase cargo space for smuggling goods - and officialdom's ineffective efforts to control the smuggling problem. The archival record addressing the San Francisco also provides a basic overview of the ship's construction: like the Santo Cristo de Burgos, it was built at the shipyard of Bagatao in Solsogón, south of Manila. It was finished in 1697 and cost nearly 62,000 pesos. The 
pitch, oil, and rigging, made of abaca hemp and gamut, cost nearly 12,000 pesos; worked iron materials, including new nails, and meals for the smiths working on them cost 7,500 pesos. ${ }^{8}$ Documents show the San Francisco's personnel and passengers had a tradition of underreporting cargo capacity and smuggling unregistered cargo to New Spain. An audit of the galleon's cargo capacity by Colonei Tomás de Endaya in 1697 apparently stated the San Francisco could hold 546 fewer pieces of cargo than it actually had available. Outraged, the resident exporters of Manila requested a new audit, arguing that space for water and supplies had been increased, thus wrongly reducing the space available for cargo. But the boatswain in charge of loading, José de Narváez, stated in 1699, during the continuing judicial inquiry, that cargo was loaded in the space reserved for water containers. The water was then stored between decks, where many of the containers broke. ${ }^{9}$

Replacing necessities with profitable cargo, and the attendant smuggling, were repeated problems. According to the account books for the Royal Treasuries of Acapulco, the Galleon General of the San Francisco, Bernardo de Hendaya, and a passenger named Seargeant Major Fernando de Iglesias each paid a fine in 1698 of 100,000 pesos for smuggling on recent galleon journeys. In 1700, General Miguel Martin, apparently a passenger on the 1700 voyage, also paid a 100,000-peso fine for smuggling. ${ }^{10}$

In 1701, gunpowder was left off the ship in order to fit more cargo bales into the hold, by packing them into the gunpowder store. The bales were mainly carrying pepper, a highly lucrative spice." A 1697 audit of the ship affirmed it could legitimately hold 3,100 bales of cargo and blocks of wax, while leaving room for water, the gunpowder store, and the ballast. A 1703 audit, however, recalculated its capacity, judging there to be additional cargo storage space available. As a result, "the supplies and water were not in their place, but exposed to the contingencies of such a long and tedious voyage, from these islands to the port of Acapulco."12 On its return from Acapulco in 1701, the San Francisco carried 60,000 pesos for the situado - the annual subsidy from the Spanish government for the Philippine colony, which was always carried in the galleon on its return voyage to Manila. ${ }^{13}$

We acquired the entire cargo manifest of the 1701 San Francisco Xavier voyage, with all the shippers' marks of each consignment - four of which fit known shippers' marks in Oregon. This cargo manifest is available as a separate appendix to the electronic version of this article and on the Oregon Historical Society website. ${ }^{14}$ The manifest is important for comparative research on Oregon's Manila galleon for several interrelated reasons. First, the same shippers frequently sent merchandise (although not necessarily the same goods) to New Spain on the annual Manila galleon for years at a stretch, especially as the trade became more consolidated due to the capital needed to undertake shipment of goods. ${ }^{15}$ Some shippers were merchants 


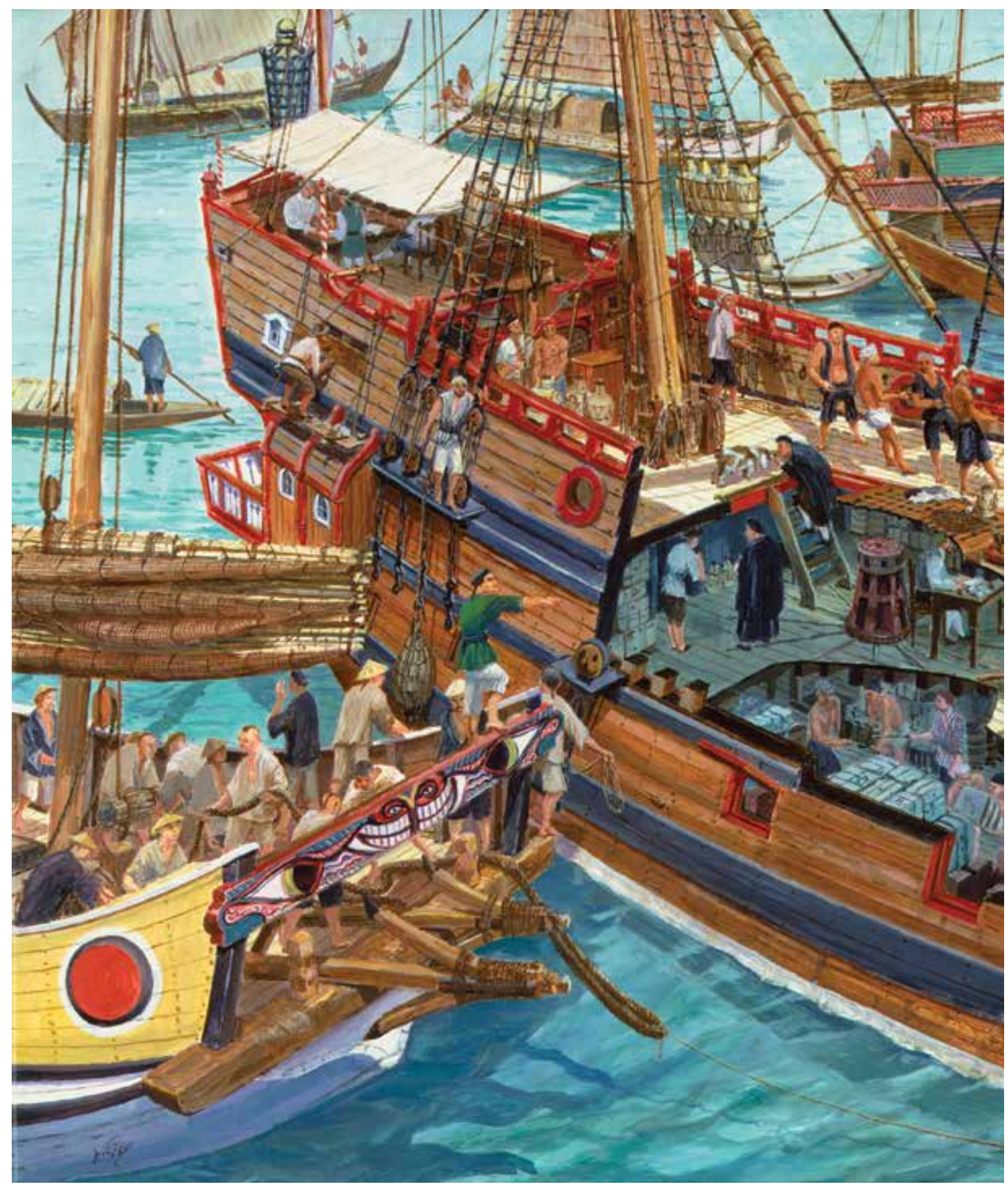

MANILA GALLEONS were built as cargo ships and were customarily heavily loaded for the trans-Pacific journey. Skilled Chinese laborers packed the barrels, especially those containing silks and other expensive goods, carefully and tightly to withstand the rigors of the voyage. This painting by Robert McGinnis illustrates the complex job of loading a galleon. 


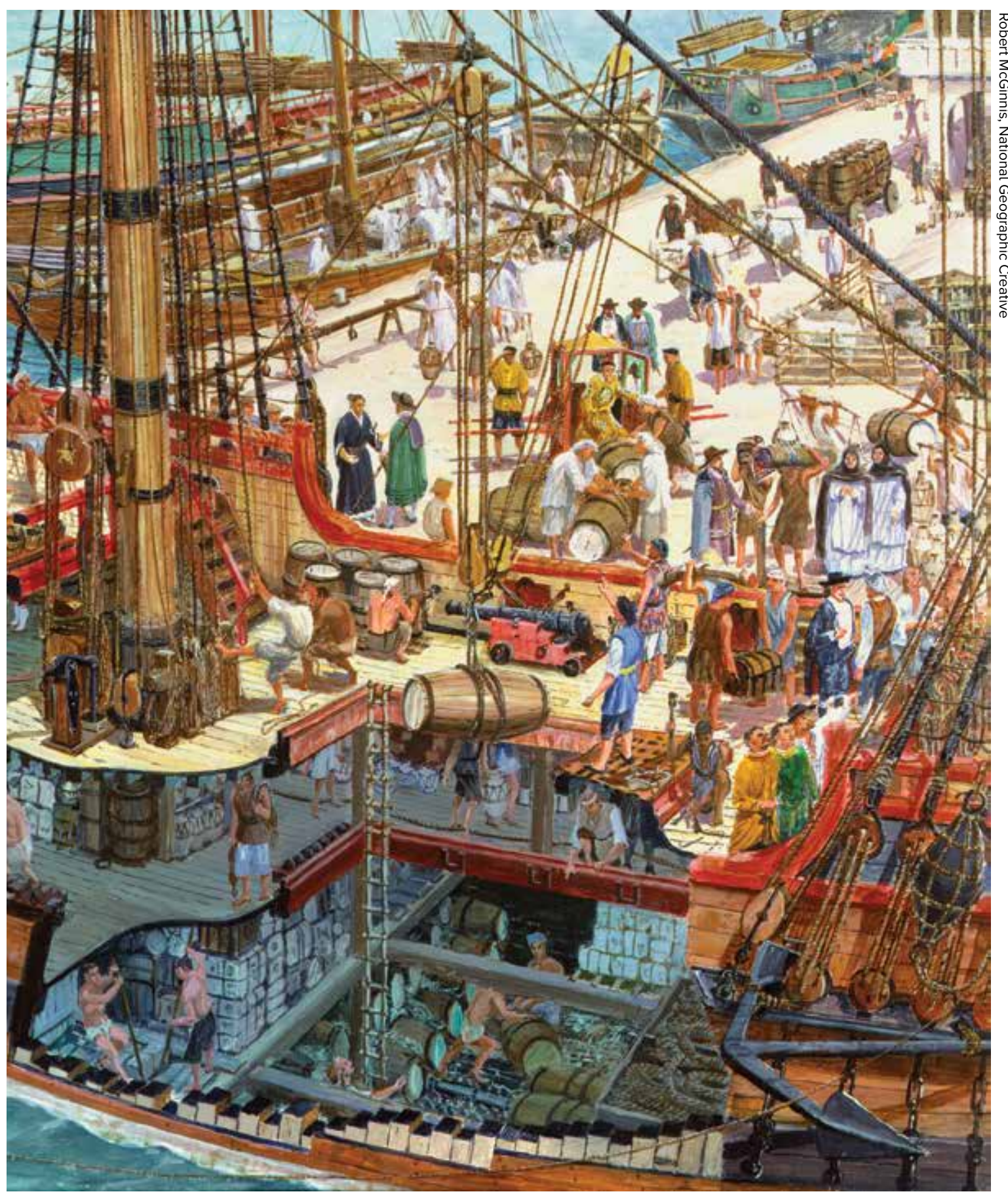


in the Philippines, others were merchants or brokers, often from New Spain or Peru, but government officials and ecclesiastics also regularly dispatched merchandise on the galleons. ${ }^{16}$ Thus, at least some shippers listed on this manifest are likely to have had consignments on the Santo Cristo of 1693 as well - only eight years before. Second, every bale when packed had the shipper's individual mark placed on it so it could be easily identified, and each shipper always used the same mark. ${ }^{17} \mathrm{It}$ is therefore probable that some of the shippers' marks on this 1701 manifest were on the Santo Cristo's 1693 manifest also, although disappointingly, none match those on the Lequeder partial manifest discussed below.

Merchandise shipped on the 1701 trip of the San Francisco Xavier consisted of the usual cargo for a Manila galleon of the period, primarily Chinese goods, which gives us a reasonable indication of what was aboard the Santo Cristo eight years earlier. The 1701 San Francisco Xavier cargo included white cotton clothes, Chinese silks, satins and damasks, petticoats and blankets, bedcovers, embroidered tapestries, Chinese fans, wooden trunks, writing desks, cups for drinking chocolate, faience (glazed ceramic ware), incense, liturgical and religious objects such as carved ivory statuettes, little boxes, "boxes with presents," combs, Chinese screens, beeswax blocks, cinnamon, and pepper. ${ }^{18}$ These latter two items, both spices, were highly valued and very lucrative.

Similarly, the cargo on the San Francisco Xavier's last trip in 1705 was as rich as ever sailed on a Manila galleon. Cook, quoting Jean de Monségur, a contemporary observer writing shortly after the galleon's departure and naturally assuming it would arrive safely, summarized it: "On his final voyage from Manila, Zabalburu embarked 500 cakes of beeswax from 10 to 14 arrobas (250 to 350 lbs. apiece, somewhere between 62 and 87 tons) valued at 150,000 piasters (pieces of eight, or dollars). Two thousand packages of silks, an equal number of bundles of diverse merchandise, gold ingots to the sum of 2,500 piasters, and a quantity of porcelain, pepper, cloves, nutmeg and aromatic drugs composed the rest of the cargo, for an estimated total value of 4,000,000."19 But the ship disappeared. In 1706, a year after the San Francisco vanished without a trace, the Viceroy of New Spain wrote that the most recent situado was overdue. Officials in Mexico had received no letters from the Philippines. Officials writing in 1708 continued to report they had heard nothing of the ship's fate. ${ }^{20}$

\section{SANTO CRISTO DE BURGOS OF 1693: CARGO KNOWN AND CONJECTURED}

We found the Archives of the Indies contains some documents relating to the cargo carried in 1693 by the Santo Cristo. Although this is the archive of record for most galleon cargo manifests, we failed to locate a manifest for the Santo 
Cristo de Burgos, either for its 1693 trip or earlier successful 1690 voyage, or the abortive 1692 trip that ended in a return to the Philippines after a major storm. ${ }^{21}$ In addition, we searched the National Archives of the Philippines in Manila and the Archivo General de la Nacíon (AGN) in Mexico City, Mexico, in hopes that cargo manifests or even partial cargo lists might have been archived there. We had no success.

Through a careful review of Spanish archival records, however, we have developed a more complete understanding of the materials carried aboard that ship and subsequently scattered along northern Oregon beaches in the late seventeenth century. A single passenger's troubles with Spanish colonial officialdom offers that glimpse of the ship's cargo on the fatal 1693 trip. Felicitous information from archival research on silver refining techniques in the seventeenth century Spanish Empire led to more information on the Santo Cristo's cargo. And by comparing the shippers' marks on beeswax blocks found in the vicinity of the Nehalem Spit with shippers' marks on the San Francisco Xavier's 1701 cargo manifest, we determined the likely identity of four shippers

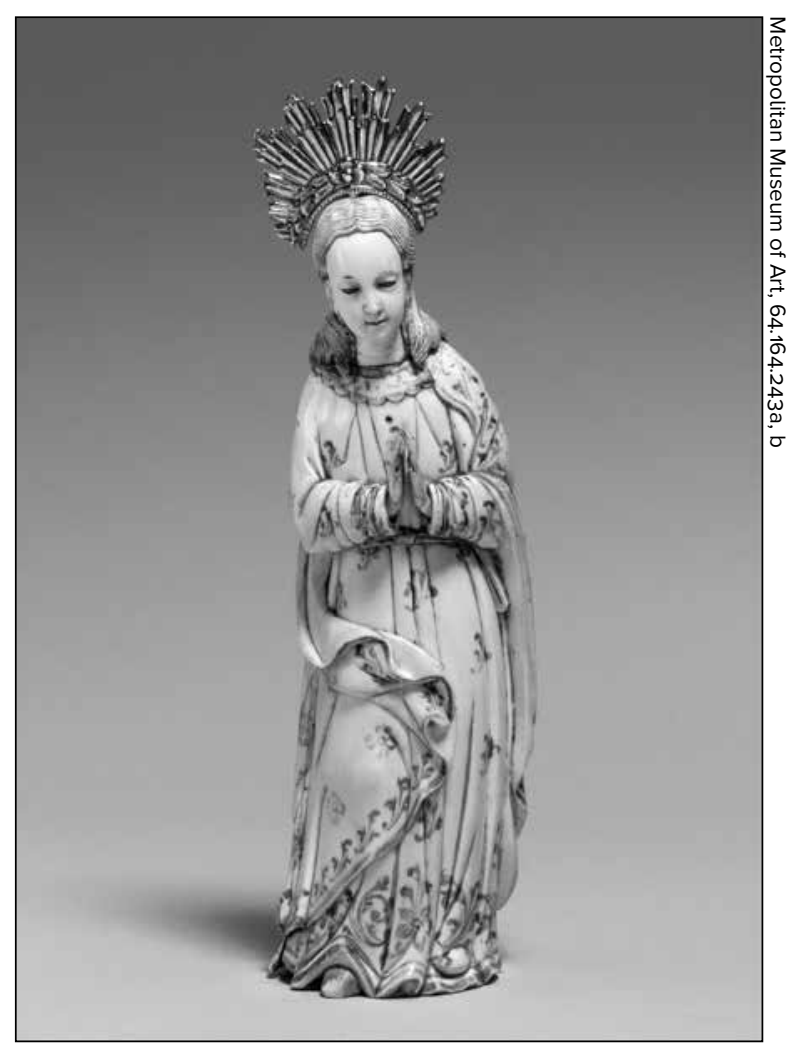

THIS EIGHTEENTH-CENTURY ivory statuette of the Virgin of the Immaculate Conception was made in the Philippines for the Manila trade. Statuettes manufactured for ecclesiastical use throughout the Americas and Europe were among the earliest Asian goods manufactured solely for global export. They were commonly included in galleon cargoes - likely including that of the Santo Cristo de Burgos.

\section{of beeswax on the Santo}

Cristo de Burgos's 1693 trip.

We were unable to locate archival records listing what else, if anything, those four shippers may have sent on the Santo Cristo in 1693.

The most direct cargo information resulted from Spanish officials' efforts to control the smuggling of Sergeant Major Pedro Lequeder y Garabalda 
of Mexico City, then residing in the Philippines. It is a complicated tale of shifting merchandise-ownership contracts, third party consignments, and government interventions. Lequeder was in persistent trouble with the Spanish administration in the Philippines for his smuggling activities. Spanish royal orders of the time reserved the Manila galleon trade only to Philippine residents. But the trade was so lucrative that enterprising merchants of New Spain would settle in the Philippines with large sums given them by Mexican residents to buy merchandise on their behalf and smuggle it back to New Spain. Lequeder was one of these Mexican brokers. He had a history of smuggling via the Manila galleon trade: by 1687, he was tangled in a lawsuit for smuggling aboard the Santo Niño y Nuestra Señora de Guía. When that galleon was forced ashore during storms and the cargo unloaded, officials discovered that Lequeder's goods had been boarded illegally to avoid paying Spanish taxes; he was ordered to pay a fine of 30,000 pesos for smuggling non-registered goods. When Lequeder did not pay, the mayor of Camarines province seized his belongings and placed him under house arrest. He, in turn, requested return of his possessions and permission to travel to Manila to defend himself in court. Officials allowed him to travel, although it seems his possessions were not identified, except some clothing. ${ }^{22}$

Another broker involved in this legal tangle, Pedro Verastagul, was found guilty and fined for defrauding the Royal Treasury by smuggling. Verastagul was the inheritor of Sargeant Major Juan de Gardaga, who boarded the Santo Cristo de Burgos in 1692 for New Spain. The ship was forced back to port at Naga by storms, and Gardaga died at the convent of San Francisco while the Santo Cristo was undergoing repairs. In his will, he appointed consignees to administer his estate; Lequeder was to collect his belongings if he should die. Thus, Lequeder inherited Gardaga's goods: a writing desk with Gardaga's personal papers, valuable possessions, clothing, and accounting books as well as Gardaga's trade goods, registered, with identifying marks, for General Antonio Nieto. Under scrutiny for smuggling, these goods - Gardaga's consigned cargo, for which Lequeder was now responsible - were properly registered as galleon cargo aboard the 1693 trip of the Santo Cristo de Burgos. They were carefully listed, and the records placed in the official archives. ${ }^{23}$

Faced with so many tangled legal disputes, the Attorney General of the Philippines, D. Juan Quijano, asked the King to issue an order that these brokers, Lequeder among them, be sent back to Mexico on the next available galleon, and meantime be prohibited from any business in the Philippines. Their cargo aboard the ship was to be embargoed. Thus, Lequeder found himself on the Santo Cristo de Burgos in 1693, along with Gardaga's goods, knowing that he would undoubtedly face charges of smuggling and thereby 
defrauding the Royal Treasury on his return to New Spain. ${ }^{24}$ He never arrived. If the Santo Cristo is determined to be the Nehalem wreck, he died on the Oregon coast, or on the voyage, of the ill-fated galleon. Luckily, the AGI contained the partial manifest listing Gardaga's consigned goods, now in the possession of passenger Lequeder. We explore that cargo list in more detail in the pages that follow.

\section{KNOWN CARGO ON THE SANTO CRISTO DE BURGOS OF 1693}

The partial cargo list for the 1693 Santo Cristo voyage reproduced on the following pages tallies Gardaga's goods in Lequeder's possession, including the shippers' marks for each consignment, which are shown next to that consignment. The majority of goods listed are cloths of different kinds, ranging from white cottons to embroidered silk with golden flowers. In addition to being a highly popular trade good in Europe by this time, Chinese silks were much in demand in New Spain (Mexico) and what is now Peru, especially after 1579, when the Manila trade began to deliver huge quantities of affordable Chinese silk to the Spanish colonies of South America. The Manila galleons were, above all, silk ships, carrying a wide array of silks, ranging from fine damask to exquisite gauzes, taffetas, flowered silks of Canton, and heavy brocade worked in glittering designs with gold and silver thread. In addition, the galleons carried other silken apparel and goods, such as skirts, cloaks, robes, and stockings; tablecloths, napkins, and bed coverings in worked silk; and heavily embroidered vestments for the Catholic clergy of New Spain. ${ }^{25}$

This partial manifest also describes a large proportion of the goods as "cottons." In part, this is a reflection of the galleon economy, which usually - as in our case as well - included fine cottons from India, including Bengal and the Malabar and Coromandel coasts. Philippine cottons were also a staple of the Manila trade, especially cotton cloth from llocos, and cotton gauzes from Cebu. Linen sheets, tablecloths, coverlets, and hammocks, manufactured throughout the Philippines, were also very popular in New Spain. ${ }^{26}$ It is necessary, however, to keep the more notorious aspects of the Manila trade in mind as well. Scholars such as William Schurz describe a widespread smugglers' tactic of placing cheap goods on the top of a bale so inspectors would not see the fine silks and other wares, such as spices, jewelry, and figurines, underneath. ${ }^{27}$ Thus, recognizing the practices of the time and Lequeder's reputation as a smuggler, it is reasonable to consider the possibility that the amount of cotton clothing reported here may be deceptive and that other, more valuable, goods could have been hidden under this merchandise, despite Lequeder's tangles with Spanish authority for smuggling activities.

The shippers' marks for Lequeder's cargo, as they appear in the original handwritten cargo list, are pictured on the following pages. Disappointingly, 
PARTIAL CARGO LIST FOR THE 1693 SANTO CRISTO DE BURGOS

Shippers' marks for each consignment item are pictured to the left of the list item.

\begin{tabular}{|c|c|c|c|c|}
\hline $\begin{array}{c}\text { SHIPPER'S } \\
\text { MARK }\end{array}$ & PACKAGE & NUMBER & MERCHANDISE & $\begin{array}{l}\text { AMOUNT/ } \\
\text { PIECES }\end{array}$ \\
\hline & Small Bundle & 2 & $\begin{array}{c}\text { Thick cotton blankets, white color, } 19 \\
\text { varas each }\end{array}$ & 116 \\
\hline & Small Bundle & $3,5,7,9$ & $\begin{array}{c}\text { Thick cotton blankets, white color, } 19 \\
\text { varas each }\end{array}$ & 100 \\
\hline & Small Bundle & 10 & $\begin{array}{c}\text { Thick cotton blankets, white color, } 19 \\
\text { varas each }\end{array}$ & 110 \\
\hline & Small Bundle & 14 & $\begin{array}{c}\text { Thick cotton blankets, white color, } 19 \\
\text { varas each }\end{array}$ & 100 \\
\hline & Small Bundle & 16,17 & $\begin{array}{c}\text { Thick cotton blankets, white color, } 19 \\
\text { varas each }\end{array}$ & 100 \\
\hline & Small Bundle & 19 & $\begin{array}{c}\text { Thick cotton blankets, white color, } 19 \\
\text { varas each }\end{array}$ & 110 \\
\hline & Small Bundle & 21,22 & Bedcovers, 2, 5 varas each & 200 \\
\hline & Small Bundle & 24 & Printed cotton fabric, 10 varas each & 125 \\
\hline & Small Bundle & 24 & Rugs of printed cotton fabric & 40 \\
\hline & Small Bundle & 26,27 & Printed cotton fabric, 11 varas each & 125 \\
\hline & Small Bundle & 33,34 & $\begin{array}{c}\text { Fine fabrics, white color, } 17 \text { and } 18 \text { varas } \\
\text { each }\end{array}$ & 55 \\
\hline & Small Bundle & $\begin{array}{c}37,38,39 \\
41,44\end{array}$ & Fine fabrics, 17 and 18 varas each & 60 \\
\hline & Small Bundle & 45 & Fine fabrics & 60 \\
\hline & Small Bundle & 47 & White cotton clothes, 19 varas each & 45 \\
\hline & Small Bundle & 48 & White cotton clothes & 50 \\
\hline & Small Bundle & 49 & White cotton clothes & 45 \\
\hline
\end{tabular}

SOURCE: Ministerio de Educación, Cultura y Deporte, Archivo General de Indias, Escribania, 442D. L.2, F. 119R-129V (Spanish list in González Research Report to La Follette, March 2016, 3-9; English translation in González Research Report Cargo Manifest \& Marks to La Follette May 2016, 1-12). 


\begin{tabular}{|c|c|c|c|c|}
\hline $\begin{array}{l}\text { SHIPPER'S } \\
\text { MARK }\end{array}$ & PACKAGE & NUMBER & MERCHANDISE & $\begin{array}{l}\text { AMOUNT/ } \\
\text { PIECES }\end{array}$ \\
\hline & Small Bundle & 52 & $\begin{array}{c}\text { Fine fabrics, white color, } 17 \text { and } 18 \text { varas } \\
\text { each }\end{array}$ & 60 \\
\hline & Small Bundle & 53,55 & White cotton clothes & 45 \\
\hline & Small Bundle & 61,64 & $\begin{array}{l}\text { Cotton clothes from Cambay, blue color, } \\
\qquad 8 \text { varas each }\end{array}$ & 140 \\
\hline & Small Bundle & 65 & $\begin{array}{l}\text { Cotton clothes from Cambay, blue color, } \\
\qquad 8 \text { varas each }\end{array}$ & 130 \\
\hline & Small Bundle & 66 & $\begin{array}{l}\text { Cotton clothes from Cambay, blue color, } \\
\qquad 8 \text { varas each }\end{array}$ & 150 \\
\hline & Small Bundle & 67 & $\begin{array}{l}\text { Cotton clothes from Cambay, blue color, } \\
\qquad 8 \text { varas each }\end{array}$ & 130 \\
\hline & Small Bundle & 68 & $\begin{array}{l}\text { Cotton clothes from Cambay, blue color, } \\
\qquad 8 \text { varas each }\end{array}$ & 50 \\
\hline & Small Bundle & 68 & $\begin{array}{l}\text { Cotton fabric from India, varied colors, } 7 \\
\text { varas length/ } \\
1 \text { vara wide }\end{array}$ & 80 \\
\hline & Small Bundle & $69,71,72,74$ & $\begin{array}{c}\text { "surcatan blancos finos de a } 19 \text { varas de } \\
\text { largo TRADUCIR" }\end{array}$ & 65 \\
\hline & Small Bundle & 76 & $\begin{array}{c}\text { Cotton clothes, white color, 6-8 varas } \\
\text { each }\end{array}$ & 119 \\
\hline & Small Bundle & 76 & $\begin{array}{c}\text { Cotton fabric from India, varied colors, } 7 \\
\text { varas each }\end{array}$ & 50 \\
\hline & Small Bundle & 78,80 & Cotton fabric from India, varied colors & 150 \\
\hline & Small Bundle & $81,82,84,85$ & White cotton clothes, 40 varas each & 23 \\
\hline & Small Bundle & 87 & White cotton clothes, 40 varas each & 24 \\
\hline & Small Bundle & $\begin{array}{l}88,89, \text { y } 90 \\
91,93,95,96\end{array}$ & White cotton clothes, 40 varas each & 23 \\
\hline & Small Bundle & $\begin{array}{l}97,98,99 \\
100\end{array}$ & White cotton clothes, 40 varas each & 24 \\
\hline
\end{tabular}




\begin{tabular}{|c|c|c|c|c|}
\hline $\begin{array}{c}\text { SHIPPER'S } \\
\text { MARK }\end{array}$ & PACKAGE & NUMBER & MERCHANDISE & $\begin{array}{c}\text { AMOUNT/ } \\
\text { PIECES }\end{array}$ \\
\hline & Small Bundle & 1,2 & White cotton clothes, 40 varas each & 23 \\
\hline & Small Bundle & $\begin{array}{c}3,4,5,6,7,8 \\
9,10\end{array}$ & Blankets from Lanquin, 100 varas each & 100 \\
\hline & Small Bundle & $\begin{array}{c}3,4,5,6,7,8 \\
9,10\end{array}$ & White cotton clothes, 40 varas each & 6 \\
\hline & Small Bundle & $\begin{array}{c}13,14,15,16 \\
\text { y } 17\end{array}$ & $\begin{array}{l}\text { Blankets from Lanquin, white color, } 11 \\
\text {-10 varas }\end{array}$ & 110 \\
\hline & Small Bundle & $\begin{array}{c}13,14,15,16 \\
\text { y } 18\end{array}$ & White cotton clothes, 40 varas each & 6 \\
\hline & Small Bundle & 18 & $\begin{array}{l}\text { Blankets from Lanquin, white color ( } 11 \\
\text { of them) }\end{array}$ & 110 \\
\hline & Small Bundle & 18 & White cotton clothes, 40 varas each & 6 \\
\hline & Small Bundle & 19 & White cotton clothes, 40 varas each & 6 \\
\hline & Small Bundle & 19 & $\begin{array}{c}\text { Blankets from Lanquin, white color, } 12 \\
\text { varas length / o,66 varas width }\end{array}$ & 100 \\
\hline & Small Bundle & 20 & $\begin{array}{l}\text { Blankets from Lanquin, white color, } 12 \\
\text { varas length / o,66 varas width }\end{array}$ & 50 \\
\hline & Small Bundle & 20 & White cotton clothes, 40 varas each & 16 \\
\hline & Small Bundle & 21 & White cotton clothes, 40 varas each & 6 \\
\hline & Small Bundle & 21 & $\begin{array}{c}\text { Blankets from Lanquin, white color, } 12 \\
\text { varas }\end{array}$ & 80 \\
\hline & Small Bundle & $22,23,25$ & $\begin{array}{c}\text { Blankets from Lanquin, white color, } 12 \\
\text { varas }\end{array}$ & 65 \\
\hline & Small Bundle & $22,23,25$ & White cotton clothes, 40 varas each & 6 \\
\hline & Small Bundle & $\begin{array}{l}26,27,28 \\
29,30\end{array}$ & White cotton clothes, 40 varas each & 22 \\
\hline & Small Bundle & 31 & Quiña silk (lbs.) & 135 \\
\hline & Small Bundle & 31 & White cotton clothes, 19 varas each & 18 \\
\hline
\end{tabular}




\begin{tabular}{|c|c|c|c|c|}
\hline $\begin{array}{l}\text { SHIPPER'S } \\
\text { MARK }\end{array}$ & PACKAGE & NUMBER & MERCHANDISE & $\begin{array}{c}\text { AMOUNT/ } \\
\text { PIECES }\end{array}$ \\
\hline & Small Bundle & 32 & Quiña silk (lbs.) & 135 \\
\hline & Small Bundle & 32 & White cotton clothes & 15 \\
\hline & Small Bundle & 36 & Quiña silk (lbs.) & 135 \\
\hline & Small Bundle & 36 & Fabrics, 17 and 18 varas each & 16 \\
\hline & Small Bundle & 34 & Quiña silk (lbs.) & 135 \\
\hline & Small Bundle & 34 & White cotton warm clothes & 16 \\
\hline & Small Bundle & 35 & Quiña silk (lbs.) & 135 \\
\hline & Small Bundle & 35 & White cotton warm clothes & 14 \\
\hline & Small Bundle & $\begin{array}{l}37,38,39 \\
40,41,42\end{array}$ & Maso silk (lbs.) & 135 \\
\hline & Small Bundle & $\begin{array}{l}37,38,39 \\
40,41,43\end{array}$ & Blankets, white color, 10 varas each & 30 \\
\hline & Small Bundle & 47 & Silk fabric, varied colors, 16 varas each & 70 \\
\hline & Small Bundle & 47 & $\begin{array}{c}\text { Fabrics from China, red color, } 6 \text { y } 7 \text { taes } \\
\text { each }\end{array}$ & 70 \\
\hline & Small Bundle & 47 & White cotton clothes, 40 varas each & 8 \\
\hline & Small Bundle & 49 & Damasks, white color, 16 varas each & 75 \\
\hline & Small Bundle & 49 & Satin, white color, 16 varas each & 19 \\
\hline & Small Bundle & 49 & White cotton clothes, 40 varas each & 6 \\
\hline & Small Bundle & 52 & Maso silk (lbs.) & 115 \\
\hline & Small Bundle & 52 & White cotton clothes, 40 varas each & 40 \\
\hline
\end{tabular}




\begin{tabular}{|c|c|c|c|c|}
\hline \multirow[t]{9}{*}{$\begin{array}{l}\text { SHIPPER'S } \\
\text { MARK }\end{array}$} & PACKAGE & NUMBE & MERCHANDISE & $\begin{array}{c}\text { AMOUNT/ } \\
\text { PIECES }\end{array}$ \\
\hline & Small Bundle & 53 & Maso silk (lbs.) & 110 \\
\hline & Small Bundle & 53 & White cotton clothes, 40 varas each & 6 \\
\hline & Small Bundle & 24 & $\begin{array}{c}\text { Cotton fabric from India, varied colors, } 7 \\
\text { varas length / nearly } 1 \text { vara width }\end{array}$ & 125 \\
\hline & Small Bundle & 24 & $\begin{array}{l}\text { Philippine fabric, made from abaca, red } \\
\text { color, } 8 \text { varas each }\end{array}$ & 13 \\
\hline & Small Bundle & 36 & Shirts and pants, white color & 300 \\
\hline & Small Bundle & 36 & $\begin{array}{l}\text { Cushions, made from Damask, gold } \\
\text { embroidered }\end{array}$ & 24 \\
\hline & Small Bundle & 36 & Silk stockings, varied colors & 250 \\
\hline & Small Bundle & 36 & Cotton fabric from India, varied colors & 25 \\
\hline & Small Bundle & 30 & Chinese silk, black color, 25 varas each & 40 \\
\hline & Small Bundle & 30 & Silk from Cambay, varied colors & 40 \\
\hline & Small Bundle & 30 & Satin, varied colors & 17 \\
\hline & Small Bundle & 30 & White cotton clothes & 4 \\
\hline & Small Bundle & 30 & Blankets from Lankin & 2 \\
\hline & Small Bundle & 31 & Wide pieces of Satin & 54 \\
\hline & Small Bundle & 31 & Satins, varied colors, 16 varas each & 32 \\
\hline & Small Bundle & 31 & White cotton clothes & 6 \\
\hline & Small Bundle & 32 & Satins from Japan & 36 \\
\hline
\end{tabular}




\begin{tabular}{|c|c|c|c|c|}
\hline $\begin{array}{l}\text { SHIPPER'S } \\
\text { MARK }\end{array}$ & PACKAGE & NUMBER & MERCHANDISE & $\begin{array}{l}\text { AMOUNT/ } \\
\text { PIECES }\end{array}$ \\
\hline & Small Bundle & 32 & Narrow pieces of Satin & 52 \\
\hline & Small Bundle & 33 & Embroidered silk, with golden flowers & 151 \\
\hline & Small Bundle & 33 & Satins, varied colors & 54 \\
\hline & Small Bundle & 33 & White cotton clothes & 6 \\
\hline & Small Bundle & 34 & $\begin{array}{l}\text { Embroidery, varied colors, } 16 \text { varas } \\
\text { each }\end{array}$ & 54 \\
\hline & Small Bundle & 34 & Chinese silk, bright color & 60 \\
\hline & Small Bundle & 34 & Chinese silk, bright color & 6 \\
\hline & Small Bundle & 34 & Blankets & 2 \\
\hline & Small Bundle & 34 & Philippine fabric, made from abaca & 1 \\
\hline & Small Box & 1 & Silk from Cambay & 6 \\
\hline & Small Box & 2 & Damasks, 16 varas each & 65 \\
\hline & Small Box & 3 & $\begin{array}{l}\text { Chinese silk, varied colors, } 16 \text { varas } \\
\text { each }\end{array}$ & 84 \\
\hline & Small Box & 4 & $\begin{array}{l}\text { Satins, floral print, golden color, } 16 \\
\text { varas each }\end{array}$ & 63 \\
\hline & Small Box & 5 & $\begin{array}{l}\text { Satins, floral print, golden color, } 16 \\
\text { varas each }\end{array}$ & 75 \\
\hline & Small Box & 6 & Damasks, yellow color, 16 varas each & 72 \\
\hline & Small Box & 7 & Satins, 16 varas each & 59 \\
\hline & Small Box & 8 & Chequered satin, 16 varas each & 63 \\
\hline
\end{tabular}




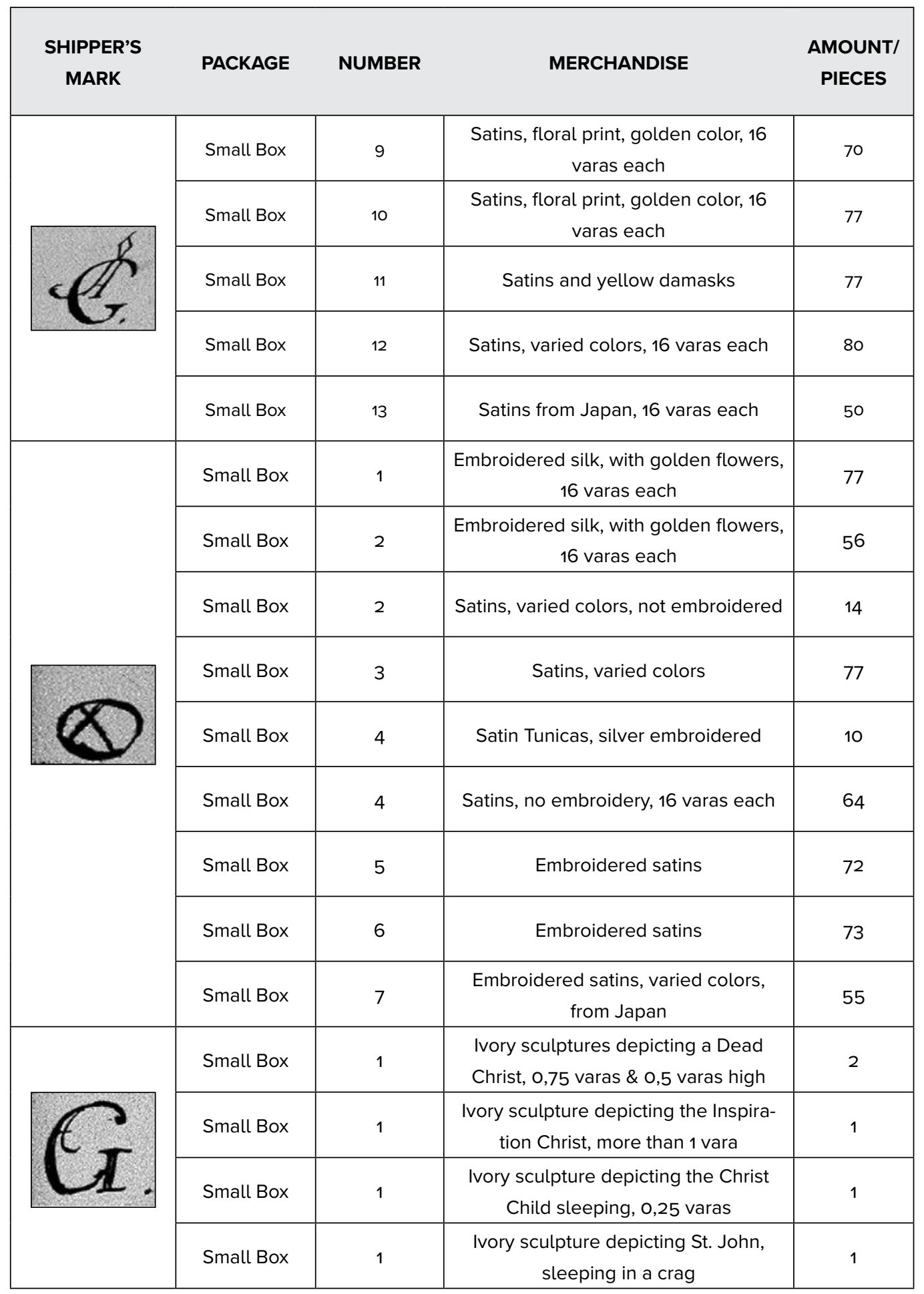




\begin{tabular}{|c|c|c|c|c|}
\hline $\begin{array}{l}\text { SHIPPER'S } \\
\text { MARK }\end{array}$ & PACKAGE & NUMBER & MERCHANDISE & $\begin{array}{l}\text { AMOUNT/ } \\
\text { PIECES }\end{array}$ \\
\hline & Small Box & 1 & $\begin{array}{c}\text { Ivory sculpture depicting the of Virgin } \\
\text { Nuestra Señora del Rosario, on a } \\
\text { base, 0,5 varas }\end{array}$ & 1 \\
\hline & Small Box & 1 & $\begin{array}{l}\text { Ivory sculpture depicting the Virgin } \\
\text { of Nuestra Señora de la Concepción, } \\
\text { 0,25 varas }\end{array}$ & 1 \\
\hline & Small Box & 1 & $\begin{array}{l}\text { Ivory sculpture depicting the Virgin } \\
\text { of Nuestra Señora Guadalupe, more } \\
\text { than } 0,5 \text { varas }\end{array}$ & 1 \\
\hline & Small Box & 1 & $\begin{array}{l}\text { Sculpture representing Santa Rosa, } \\
\text { 075 varas }\end{array}$ & 1 \\
\hline & Small Box & 1 & $\begin{array}{l}\text { Ivory sculpture depicting the Virgin } \\
\text { Nuestra Señora del Rosario, 0,33 } \\
\text { varas }\end{array}$ & 1 \\
\hline & Small Box & 1 & Stockings, white and varied colors & 180 \\
\hline & Small Box & 1 & Stockings, white color & 50 \\
\hline & Small Box & 2 & $\begin{array}{l}\text { White paper fans, with black and } \\
\text { golden ribs }\end{array}$ & 720 \\
\hline & Small Box & 2 & $\begin{array}{l}\text { White paper fans, with black and } \\
\text { golden ribs }\end{array}$ & 120 \\
\hline & Small Box & 2 & $\begin{array}{l}\text { White paper fans, with black and } \\
\text { golden ribs }\end{array}$ & 405 \\
\hline & Small Box & 2 & $\begin{array}{l}\text { White paper fans, with black and } \\
\text { golden ribs }\end{array}$ & 130 \\
\hline & Small Box & 2 & $\begin{array}{l}\text { White paper fans, with snake shaped } \\
\text { ribs }\end{array}$ & 35 \\
\hline & Small Box & 2 & $\begin{array}{l}\text { White paper fans, with black and } \\
\text { golden ribs }\end{array}$ & 40 \\
\hline & Small Box & 3 & $\begin{array}{l}\text { White paper fans, with golden and } \\
\text { enamel ribs }\end{array}$ & 1050 \\
\hline & Small Box & 3 & $\begin{array}{l}\text { White paper fans, with black and } \\
\text { golden ribs }\end{array}$ & 265 \\
\hline & Small Box & 4 & $\begin{array}{l}\text { White paper fans, with green and } \\
\text { purple enamel ribs }\end{array}$ & 1290 \\
\hline
\end{tabular}




\begin{tabular}{|c|c|c|c|c|}
\hline $\begin{array}{l}\text { SHIPPER'S } \\
\text { MARK }\end{array}$ & PACKAGE & NUMBER & MERCHANDISE & $\begin{array}{l}\text { AMOUNT/ } \\
\text { PIECES }\end{array}$ \\
\hline & Small Box & 5 & $\begin{array}{l}\text { White paper fans, with golden and } \\
\text { enamel ribs }\end{array}$ & 918 \\
\hline & Small Box & 5 & $\begin{array}{l}\text { White paper fans, with green and } \\
\text { purple enamel ribs }\end{array}$ & 220 \\
\hline & Small Box & 5 & White paper fans, with golden ribs & 45 \\
\hline & Big Box & 1 & Blankets, white color, 12 varas each & 2 \\
\hline & Big Box & 1 & Silk bed sheets, with golden flowers & 20 \\
\hline & Big Box & 1 & $\begin{array}{l}\text { Fabrics from Cantón, silver and green } \\
\text { color }\end{array}$ & 5 \\
\hline & Big Box & 1 & Tableclothes & 2 \\
\hline & Big Box & 1 & Napkins & 18 \\
\hline & Big Box & 1 & $\begin{array}{l}\text { Ivory sculpture depicting a Saint, with } \\
\text { an ebony cross, } 0,66 \text { varas }\end{array}$ & 1 \\
\hline & Big Box & 1 & Fabrics, white color & 4 \\
\hline & Big Box & 1 & $\begin{array}{c}\text { Cotton clothes, on the account of my } \\
\text { goodfather, who is in Leyte, } 16 \text { varas } \\
\text { each }\end{array}$ & 1 \\
\hline & Big Box & 1 & Blue blanket & 1.5 \\
\hline & Big Box & 1 & $\begin{array}{l}\text { Silk cinctures, on the account of Cris- } \\
\text { tóbal de León, chaplain of the Santa } \\
\text { Misericordia }\end{array}$ & 150 \\
\hline & Big Box & 1 & Silk cinctures (varas) & 45 \\
\hline & Big Box & 1 & $\begin{array}{l}\text { Silk ribbons, varied colors, on the } \\
\text { account of Cristóbal de León, chaplain } \\
\text { of the Santa Misericordia (varas) }\end{array}$ & 263 \\
\hline
\end{tabular}




\begin{tabular}{|c|c|c|c|c|}
\hline $\begin{array}{l}\text { SHIPPER'S } \\
\text { MARK }\end{array}$ & PACKAGE & NUMBER & MERCHANDISE & $\begin{array}{l}\text { AMOUNT/ } \\
\text { PIECES }\end{array}$ \\
\hline & Small Box & 1,6 & $\begin{array}{c}\text { Sacks of musk, on the account of } \\
\text { Alonso de los Ángeles }\end{array}$ & 6 \\
\hline & Small Box & & White paper fans, with black ribs & 360 \\
\hline & Small Box & & Writing paper from China & 7 \\
\hline & Small Box & & White paper fans, with black ribs & 25 \\
\hline & Small Box & & White paper fans, with red ribs & 10 \\
\hline & Small Box & & $\begin{array}{l}\text { White paper fans, with black and } \\
\text { shaped ribs }\end{array}$ & 10 \\
\hline & Small Box & & White paper fans, with black ribs & 10 \\
\hline & Small Box & & Small fans, painted, with black ribs & 10 \\
\hline & Small Box & & Small withe fans, with black ribs & 10 \\
\hline & Small Box & & $\begin{array}{l}\text { White paper fans, with black and } \\
\text { golden ribs }\end{array}$ & 5 \\
\hline & Small Box & & $\begin{array}{l}\text { White paper fans, with golden and } \\
\text { enamel ribs }\end{array}$ & 30 \\
\hline & Small Box & & $\begin{array}{l}\text { White paper fans, with green and } \\
\text { purple enamel ribs }\end{array}$ & 40 \\
\hline & Small Box & & Crockery bowls for drinking chocolate & 12 \\
\hline & Small Box & & Crockery bowls for consommé & 12 \\
\hline & Small Box & & $\begin{array}{l}\text { Small crockery with its cover and } \\
\text { plate, with golden decorations }\end{array}$ & 1 \\
\hline & Small Box & & Fabrics, red color & 3 \\
\hline & Small Box & & $\begin{array}{l}\text { Satin embroidered bedcover, lined } \\
\text { with red fabric, with silk fringes }\end{array}$ & 1 \\
\hline
\end{tabular}




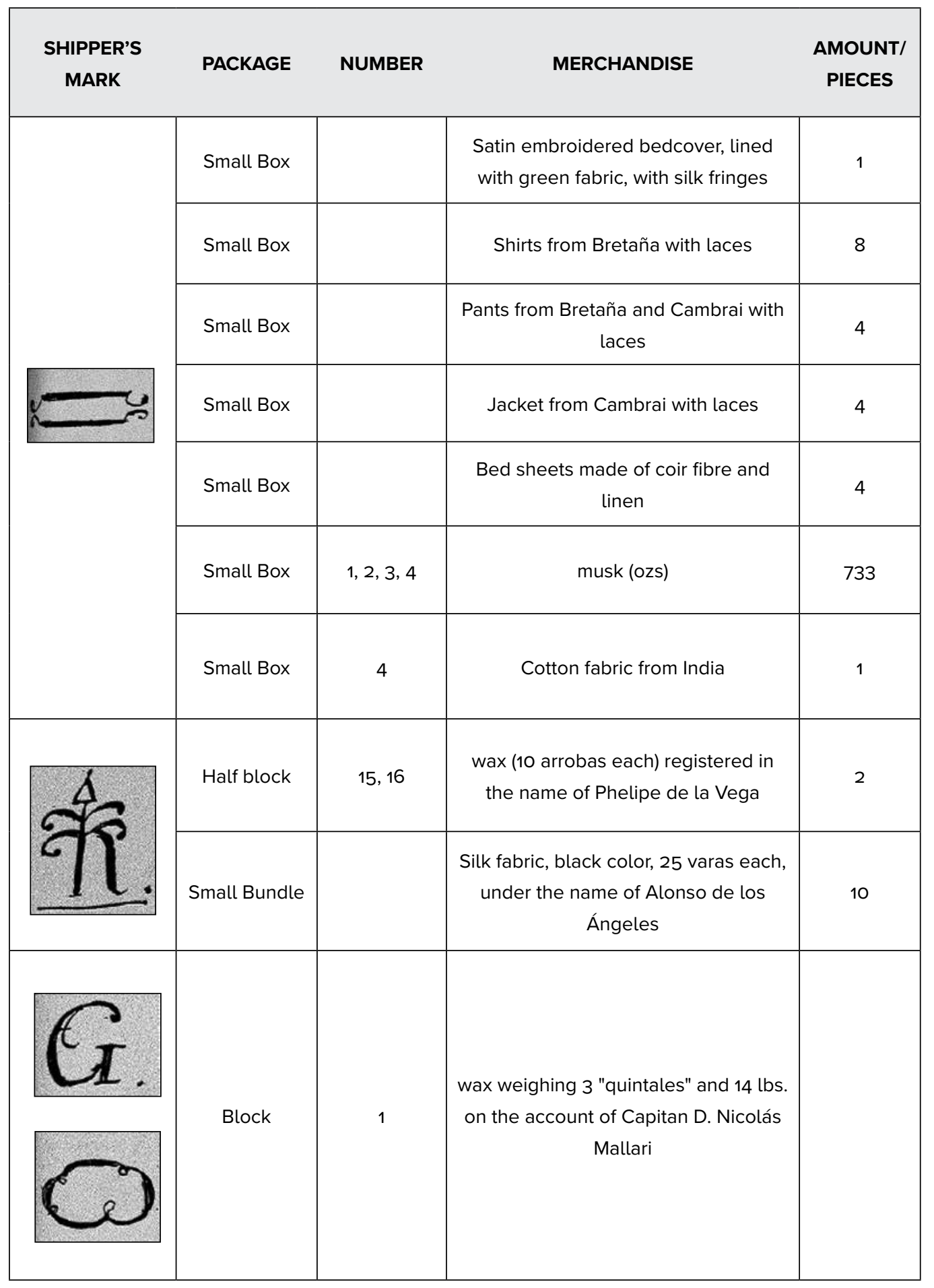


these specific shippers' marks - undoubtedly representing only a small subset of the identification marks found on cargo packages aboard the Santo Cristo - do not match any marks on beeswax blocks so far reported in Oregon.

\section{KNOWN SHIPPERS' MARKS ON NEHALEM BEESWAX BLOCKS}

Individual merchants who were shipping beeswax carved their signature marks onto the blocks to differentiate them from other merchants' goods that would be in the galleons' holds. Shippers' marks have been recognized on beeswax that washed ashore on the Oregon coast, with many reproduced in publications. Don Marshall's popular book, Oregon Shipwrecks, provides the most comprehensive set. ${ }^{28}$ Marshall's images are based on recollections of those who found chunks of beeswax or on drawings they did at the time. Sometimes missing fine-grained details, these images nonetheless show the basic features of several key marks, identifying the merchants who originally shipped those blocks. Few of the chunks (and thus the shippers' marks) are known to be extant today, but there is a well-preserved fragment in the Tillamook Pioneer Museum in Tillamook, bearing shipper's mark numbered twenty-two on Marshall's list.

Several of the merchants who shipped goods aboard the doomed Santo Cristo de Burgos voyage of 1693 also appear to have had goods aboard the successful 1701 San Francisco Xavier trip, which followed the same North Pacific route. There are four shippers from the 1701 journey whose shipping marks appear to match, or nearly match, four from the known Nehalem marks. On the 1701 San Francisco Xavier trip, these four shippers were sending not beeswax to New Spain, but primarily fabrics. This was a common phenomenon, as shippers frequently varied merchandise on different trips due to availability of goods, prices, and customer needs. Four shippers' marks as found on the 1701 San Francisco Xavier manifest are pictured on the following pages with a summary of the goods shipped by that merchant in 1701. On the facing page for comparison is a set of Nehalem marks published in the Oregonian in 1915. ${ }^{29}$ The Santo Cristo in 1693 appears to have carried a cargo similar to the items enumerated for these four shippers, as suggested by the Lequeder partial manifest.

\section{MERCURY ON THE SANTO CRISTO DE BURGOS OF 1693}

In addition to the trade goods, the Santo Cristo de Burgos carried a cargo of liquid mercury, also known as quicksilver. Mercury was an essential ingredient for the refining process used by the Spanish at its lucrative mines in South America. Silver was the wealth that fueled the Spanish Empire, especially the rich silver mines of Mexico and South America - most especially the 


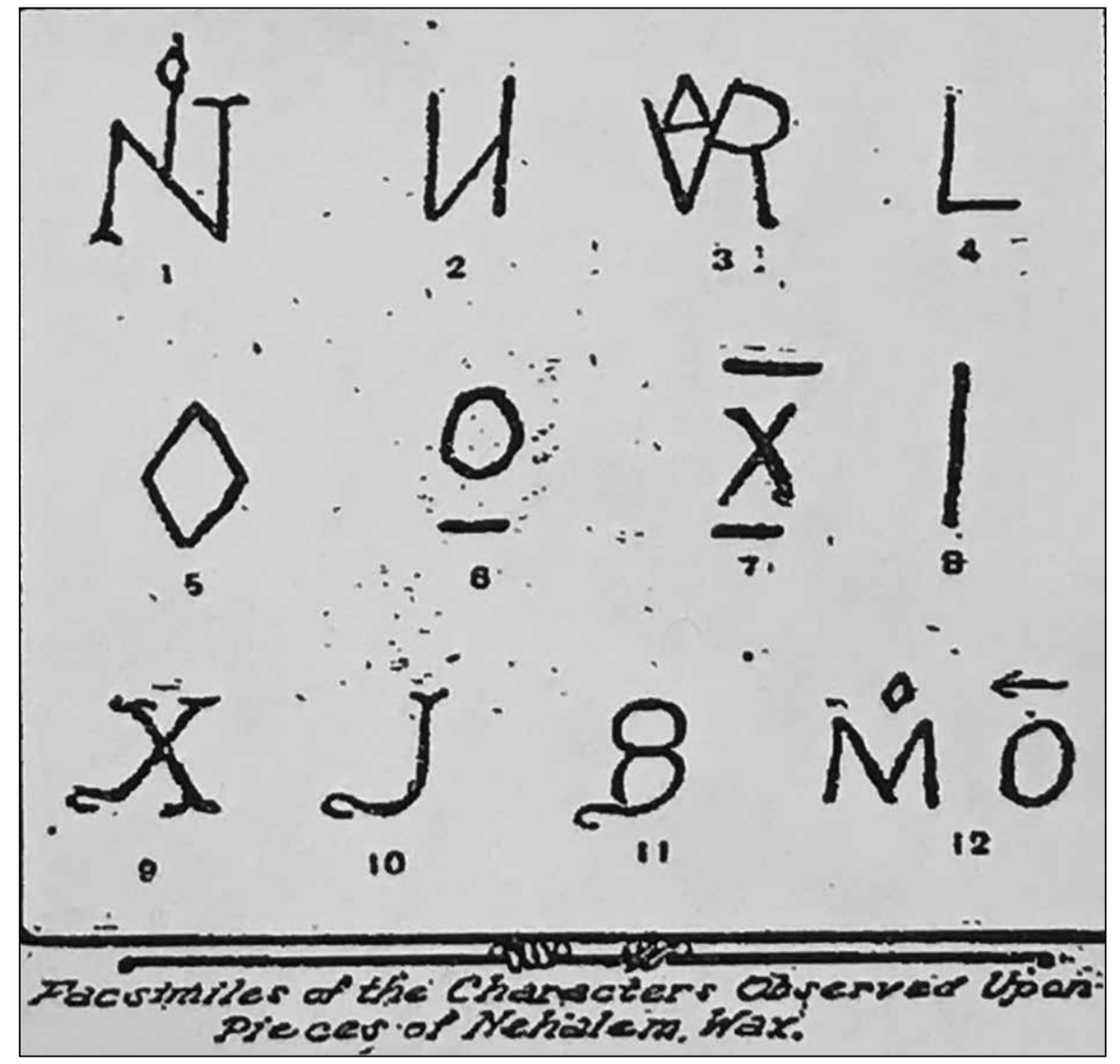

THIS TABLE OF SYMBOLS - "Facsimiles of the Characters Observed Upon Pieces of Nehalem Wax" - was published by the Oregonian in 1915. The "characters" are now known to be Spanish colonial shippers' marks found on blocks of beeswax discovered in and around Nehalem Bay. More comprehensive tables of symbols recovered from beeswax are found in such sources as Don Marshall's Oregon Shipwrecks, although in many cases, the original blocks of beeswax can no longer be located. 

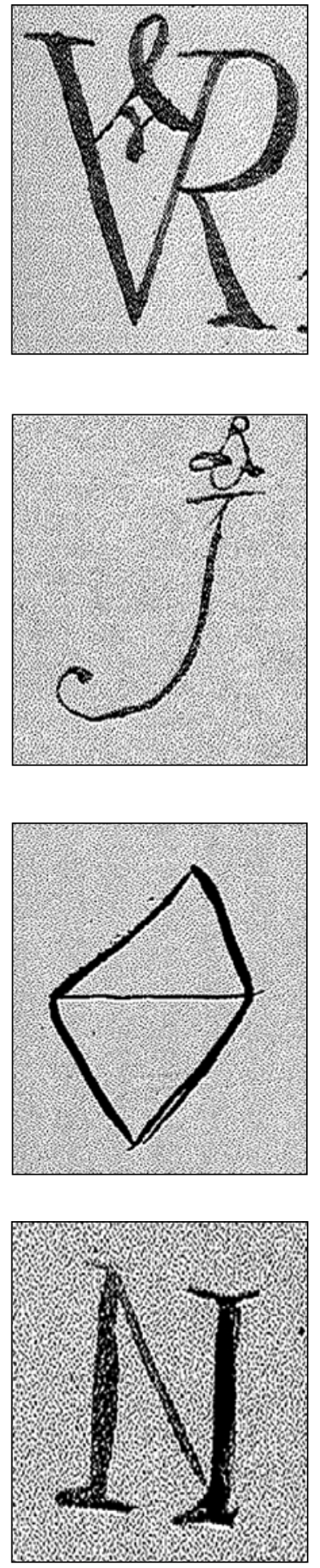

CAPTAIN FRANCISCO MANUEL DE YRRASAGORIA'S shipper's mark, pictured to the left, as recorded in the 1701 San Francisco Xavier manifest for the trip from Manila to Acapulco. The ship manifest identified Yrrasagoria's goods as white cotton clothes and fabrics. This mark closely matches the third mark on the chart on the facing page, which was found on pieces of beeswax found on the beaches of Nehalem. It also closely matches figure five in Don Marshall's Shipwrecks of Oregon. (Ministerio de Educación, Cultura y Deporte, Archivo General de Indias, Filipinas,214,N.1,F.58r.)

CAPTAIN JOSEPH DE OZCORTA'S shipper's mark is pictured here, as recorded in the 1701 San Francisco Javier manifest for the trip from Manila to Acapulco. The ship manifest documented that Ozocorta's bundle of goods contained white cotton clothes and blankets from China. This mark is similar to the tenth mark on the chart on the facing page. It also closely matches figure twelve in Don Marshall's Shipwrecks of Oregon. (Ministerio de Educación, Cultura y Deporte, Archivo General de Indias, Filipinas, 214,N.1,F.110v.)

\section{SERGEANT MAJOR FRANCISCO DE MAYA Y TORRES'S} shipper's mark on the on the 1701 San Francisco Xavier manifest, pictured to the left, contained white cotton clothes, blankets from China, silks, blankets, fabrics, writing desks, and one screen. This mark is simliar to the fifth mark on the chart on the facing page and closely matches figure seven in Don Marsall's Shipwrecks of Oregon. (Ministerio de Educación, Cultura y Deporte, Archivo General de Indias, Filipinas, 214,N.1,F.103r.)

GENERAL JUAN DE ECHEVARRÍA'S shipper's mark, as recorded on the 1701 San Francisco Xavier manifest, listed Echevarria's goods as towels and blankets from China. His mark, pictured here, is not shown on the table on the facing page, but it very closely matches figure twenty in Don Marshall's Shipwrecks of Oregon. (Ministerio de Educación, Cultura y Deporte, Archivo General de Indias, Filipinas, 214, N.1, F.133r.) 
Cerro Rico mine in Potosí, Bolivia. But silver in bulk could only be obtained by refining ground silver ore with highly poisonous mercury, as the Spanish were well aware. In an early Spanish treatise on metallurgy, Alonso Alvaro Barba (b. 1569), described mercury and its importance to the Spanish empire:

Quicksilver is a Mineral very well known, of a liquid substance, and fluid like water; it is naturally viscous, very subtil, and abounds in humidity, whence it obtains the qualities of being very heavy, and shining bright, and of being very cold... [Mercury] is notoriously known to be poyson... There was very little use or consumption of Quicksilver before the beginning of this new Silver age in the world. . . it is incredible, how great a quantity is consumed by the Founders of Mettals of this Kingdom: for if the abundance of Silver that hath gone out of this Kingdom, hath filled the world with riches and admiration; by it may be estimated the consumption and loss of Quicksilver, which after a most extravagant expence thereof at first, being now by good experience regulated within terms of moderation, is found to be equal in weight to the Silver extracted. ... They began to register the Quicksilver that came to Potosi upon the Kings accompt, in the year 1574; and from that time till 1640, there had been received of it upwards of 204600 Quintals, besides a vast quantity irregularly brought in upon other accompts. ${ }^{30}$

As this excerpt makes clear, the Spanish searched intensively for sources of mercury, both in the Americas and in Europe. This need for mercury directly impacted the galleon trade, including the Santo Cristo de Burgos.

The amalgamation method of silver refining required adding mercury and other regents to the ore. The two elements reacted by forming an amalgam, from which the refined silver was extracted by heating. This method was developed by Seville native Bartolomé de Medina in the mid sixteenth century and was widely used in Spanish silver mining from about 1560 onwards. ${ }^{31}$ The only limitation on its use was the mercury scarcity in New Spain, which led to price fluctuations and variations in quality of the available mercury. European mines (such as that at Almadén) did not produce sufficient mercury to meet the demand. This problem was exacerbated by the Spanish Crown's inconsistent payments to the German licensees of the Almadén mine in the early to mid seventeenth century; when the German investment at Almadén subsequently collapsed and the Spanish Crown took over the mine, poor management reduced the flow of mercury even further. ${ }^{32}$

The Crown responded to the acute need for mercury by importing it from other European countries, such as Slovenia, and increasing production at the Peruvian mercury mine of Santa Barbara at Huancavelica. The Crown had appropriated Huancavelica as a Crown possession in 1570 and operated it for centuries to provide mercury for the silver refining process. 


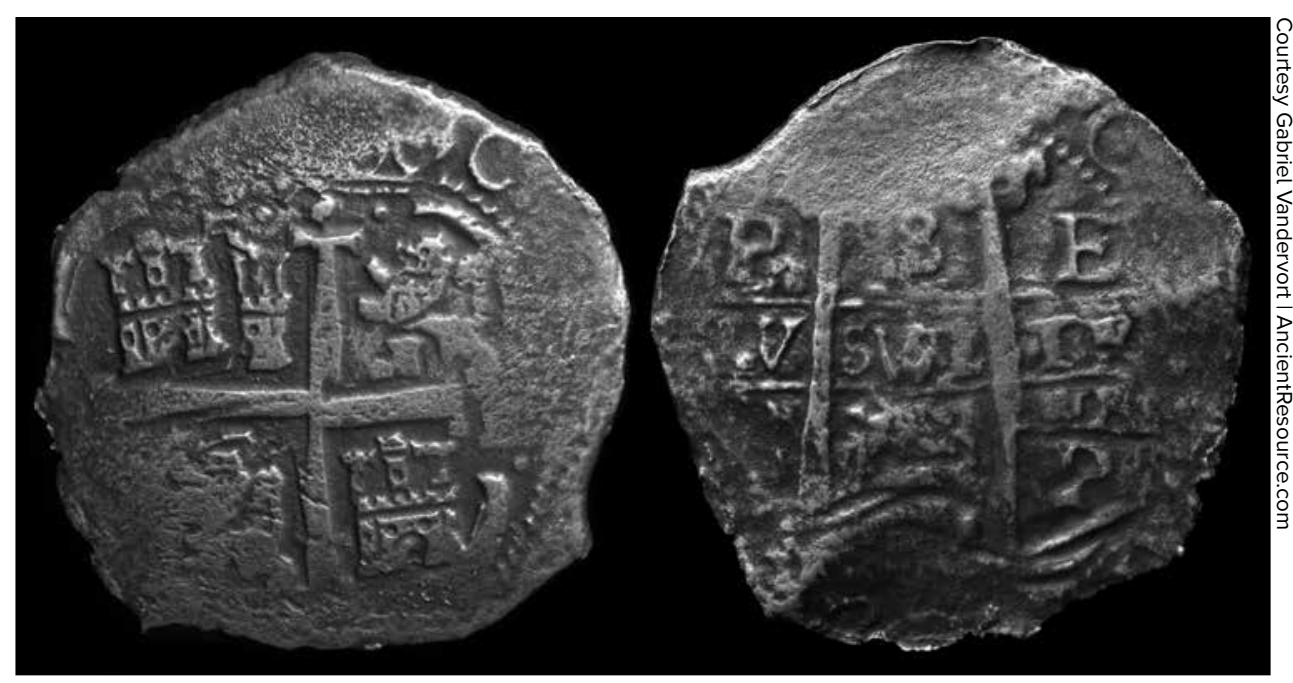

THIS SPANISH "PIECE OF EIGHT” from the wreck of the Consolacion was minted in Potosí, Bolivia in 1673. Spanish silver, especially from the Cerro Rico mine in Potosí, fueled the Manila trade. Silver refining required mercury, which Spain frequently imported from China and other regions. Chinese mercury was shipped to New Spain on Manila galleons, including the Santo Cristo de Burgos.

It was probably the most prolific source of mercury for the Crown during the colonial era. ${ }^{33}$ Nevertheless, supply problems continued, and the Spanish Empire frequently, though inconsistently, turned to China to supply the lack, transporting the mercury to New Spain via Manila galleon. The Council of the Indies in 1584 first considered the proposal to import Chinese mercury, since it could be purchased at reasonable prices. ${ }^{34}$

The proposals for a stable mercury import program did not come to fruition for various political, economic, and bureaucratic reasons, even during the Almadén mercury mine crisis in the years 1630 to 1645, and despite desperate need on the part of the silver mining operations in New Spain. The Crown never did establish a permanent trade arrangement to supply New Spain with mercury from China. Only occasionally did Spanish officials manage to purchase Chinese mercury. In 1691, the annual Manila galleon successfully delivered 1.5 quintales (hundredweight) of mercury to New Spain. ${ }^{35}$

This small success encouraged colonial administrators to look further into opportunities to acquire and ship Chinese mercury. In 1692, the Viceroy of New Spain, Don Gaspar de la Cerda Silva Sandoval y Mendoza, 8th Conde de Galve, explained to the Council of the Indies that the lack of mercury was affecting the production of silver in New Spain's mines. He again sought to purchase mercury from China, a major mercury producer, and secured the support of 
the Philippines Governor, Fausto Cruzat y Góngora Rada, for the effort. The Treasury of Mexico sent 12,000 pesos to Manila to buy 100 quintales of mercury for shipment to New Spain. Ultimately, the governor could only purchase 53 quintales. Archival records indicate that this load of mercury was shipped on the Santo Cristo de Burgos of 1693. ${ }^{36}$ The mercury was shipped as a liquid (as quicksilver), poured into leather bags, with sometimes one, but often two or three full bags placed into a small wooden barrel. ${ }^{37}$ A quintal was a Spanish measure weighing approximately 46 kilograms, or 101 U.S. pounds. ${ }^{38}$ Thus, 53 quintales of mercury equals about 5,374 pounds, approximately 2.5 tons. This liquid mercury was lost with all the other cargo on the Santo Cristo de Burgos.

In 1695, Cruzat acknowledged the 1693 royal decree about the desperate need for mercury and receipt of 60,000 pesos, of which 12,000 was earmarked for purchase of the mercury from China. As he had only been able to buy 53 quintales of mercury, spending 3,222 pesos, he returned the remaining funds to the Treasury. The governor explained he could purchase no more mercury, because he had no ship available to travel to Canton. The ship that had carried the 53 quintales of mercury from China to Manila in 1693, prior to its transfer to the Santo Cristo, was still in Manila, waiting for the Santo Cristo to return with the money brought by sale of the mercury in New Spain. The ship had been waiting for two years, and, the governor added, "We hope that God will bring the ship [Santo Cristo de Burgos] safety [sic] to this port." ${ }^{39}$

The Spanish shipped quicksilver via galleons occasionally at other times as well. Although the Santo Cristo's loss of 2.5 tons of mercury was a significant disaster, it pales compared to the wreck of the two so-called "quicksilver galleons" in Samaná Bay, Dominican Republic. The Nuestra Señora de Guadalupe and the Conde de Tolosa set sail from Cadiz, Spain, for Veracruz, Mexico, in 1724. Both were large ships of more than 1,000 tons each, which was necessary to carry the cargo: Guadalupe carried 250 tons of quicksilver, and Tolosa 150 tons. But on August 24, a hurricane struck. The Tolosa rode out most of the storm at the mouth of Samaná Bay but was ultimately pushed into the bay, beaten against shoals, and finally wrecked on a coral reef. Of more than 600 passengers and crew, fewer than 40 survived, and the cargo of mercury, spilled from its shipping casks, mostly seeped through the wreck and ultimately dripped onto the seabed. By contrast, the Guadalupe grounded on a sandbank. The 250 tons of mercury it was carrying held the ship upright, so it rode out the storm, and about 550 of the 650 people aboard reached land. The mercury remained, and still remains, in the hold of the wrecked ship. When Caribe Salvage, with permission of the Dominican Republic, came to salvage the wrecks in 1979, they located the Tolosa by the globules of mercury laying in the remnants of small wooden casks stored in rows near the bottom of 
the ship's hull, among the shipwreck's timbers..$^{40}$ This may be true for the recovery of the Santo Cristo as well.

\section{AFTERLIFE OF THE SANTO CRISTO'S CARGO}

At this time, there are no known full cargo manifests for either the San Francisco Xavier of 1705 or the Santo Cristo de Burgos of 1693. Now that the Santo Cristo is the favored candidate for the Oregon Beeswax Wreck, the failure to find a complete cargo list for the 1693 voyage is particularly frustrating. Nevertheless, there is considerable information regarding the goods carried by that ship. The cargo consigned to Pedro Lequeder is representative and gives a glimpse into the types of goods the Santo Cristo was bringing to Acapulco. The fuller cargo list from the San Francisco Xavier of 1701 provides additional clues. Shippers' marks on the beeswax recovered in Oregon seem plausible matches with four marks available in the existing archival record, from the San Francisco Xavier of 1701, and more matches may be identified in time. Resulting from extensive archival searches in several locations, these are the principal known written clues available to researchers of the remarkably rich and diverse cargoes that both ships carried on their final, but unsuccessful, efforts to reach the waiting merchants and colonists of New Spain and other markets farther afield.

Successful archaeological investigation of the wreck, should the underwater portions be located, might provide additional details about the cargo and perhaps the weaponry and storage supplies on board as well. If quicksilver is located in the vicinity of the wreckage, it would provide a strong diagnostic clue that the wreck is most likely the Santo Cristo de Burgos of 1693. In our searches, we found no original or secondary sources in any archive or other document mentioning that the San Francisco Xavier of 1705 carried mercury as part of its cargo. More exhaustive research would be valuable to finalize this preliminary conclusion.

Mercury is a very poisonous metal, as was known to the colonial Spanish. But its toxicity is much better understood now, especially its effects on marine ecosystems, which is of immediate importance to Oregon if the Santo Cristo is identified as the Nehalem wreck. A sketch of the effects of mercury worldwide will help outline the nature of the problem Oregon now faces as a result of the galleon wreck.

The current extent of mercury in the oceans due to human release of the metal into the atmosphere, especially through the burning of fossil fuels, is of great concern worldwide. Coal and oil-burning power plants release mercury into the air, where it falls to earth and the sea in rain and snow. Mercury in the oceans has risen by at least a factor of three from pre-human levels. Marine soil microorganisms convert the recently airborne 
mercury into its most toxic form for humans and wildlife: methylmercury. This form of mercury accumulates in the tissues of humans and other creatures, becoming more concentrated as it moves up the food chain. At high enough levels, it causes neurological damage. ${ }^{41}$

Of the several chemical forms mercury can take, the least harmful is elemental mercury, also known as quicksilver. When atmospheric mercury falls into the sea, more than half is "reduced" to that less harmful elemental form, most likely by mercury-reducing bacteria, especially in coastal and nearshore zones. But much atmospheric mercury is methylated - and rendered much more harmful to the food chain and living creatures, including humans - by anaerobic bacteria and other microorganisms. ${ }^{42}$

Although elemental mercury (quicksilver) does not regularly enter the ocean in large quantities, it does happen occasionally, the wrecks of the Tolosa and the Guadalupe in Samaná Bay being perhaps the most spectacular example. When salvors came to the wrecks nearly 250 years later, some of the quicksilver was still there, laying in globules among the remains. The methylation rates for elemental mercury in seawater are often slow, for reasons not well understood. It occurs at varying speeds depending on ocean depth, dissolved oxygen levels, and the presence of the marine bacteria that apparently cause it. ${ }^{43}$ As part of its cycling process, elemental mercury is also re-oxidized to the air, where it can again be re-emitted to the ocean, and it can also be buried in deep-sea marine sediments when it binds to particulates in the water. Burial effectively removes it from the biologically active ocean cycle. ${ }^{44}$

Thus, if the Santo Cristo is ultimately determined to be the Nehalem wreck, the approximately 2.5 tons of mercury lying in its holds off the Oregon coast, or scattered around on the seabed nearby, constitute an organic biohazard, as the quicksilver is likely to eventually methylate and become highly toxic. Rates and pathways to methylation depend on the specific nearshore environment. ${ }^{45}$ Elemental mercury in seawater is often fairly stable, however, so removal can be somewhat easily accomplished, if the mercury has not been covered in marine sediments by now or strewn across the seafloor. Testing of nearshore sediments and shellfish in the wreck area might give clues to its location as well as indicators of the extent of methylation.

Overall, the available Spanish colonial record provides a partial inventory of the cargo of the 1693 Santo Cristo and a complete picture of the cargo from the 1701 San Francisco Xavier. These manifests invite comparison with known and as-yet-undiscovered components of the archaeological record relating to Oregon's galleon wreck. It is our hope that these new records on the cargoes of both ships might bring clarity to research on the wreck for many years to come. 
At minimum, these archival facts regarding the galleon cargoes provide us all with a broader appreciation of the riches carried by the "treasure ships of the Pacific" via the Manila trade. When one unfortunate ship drifted from its route to Acapulco and came crashing ashore in the vicinity of Nehalem Spit, it forever affected the history and the peoples of the Oregon coast. Its most visible cargoes - beeswax blocks and Chinese porcelains - have long defined the wreck for Oregonians and continue to do so to this day. They have also been instrumental in providing archaeologists and other researchers the means to successfully begin unraveling the galleon's history and fate.

\section{NOTES}

This research was only possible with help from staff at the Archives of the Indies in Seville, Spain; the National Archives of the Philippines in Manila, the Archivo General de la Nación of Mexico in Mexico City, and the Archivo General del Estado de San Luis Potosí. The authors are grateful to Esther González Pérez, researcher at the Archives of the Indies of Seville, whose work documented the cargos of both the Santo Cristo de Burgos and the San Francisco Xavier, and provided identification of some shippers whose marks match those on beeswax blocks recovered at Nehalem. We also thank the translators Victoria Stapells and Trágora Traducciones of Granada, Spain. Rafael Castañeda Garcia of Mexico City and Rose Marie Mendoza, researcher at the National Archives of the Philippines in Manila provided additional critical research on documents relating to the Santo Cristo De Burgos.

1. See Cameron LaFollette and Douglas Deur, "Views Across the Pacific: The Galleon Trade and Its Traces in Oregon," in this issue of the Oregon Historical Quarterly, 119:2 (Summer 2018): 160-91; and Scott Williams, Curt D. Peterson, Mitch Marken and Richard Rogers, "The Beeswax Wreck of Nehalem: A Lost Manila Galleon," in this issue of the Oregon Historical Quarterly, 119:2 (Summer 2018): 192-209.

2. William Lytle Schurz, The Manila Galleon (New York: E.P. Dutton \& Co., 1939, 1959), 69; Shirley Fish, The Manila-Acapulco
Galleons: The Treasure Ships of the Pacific (Central Milton Keynes, U.K.: AuthorHouseUK, 2011), 279; Warren Cook, Flood Tide of Empire: Spain and the Pacific Northwest, 1543-1819. (New Haven: Yale University Press, 1973), 33; A.J. Schulte, "Altar Candles." in The Catholic Encyclopedia (New York: Robert Appleton Company, 1907), New Advent, http://www. newadvent.org/cathen/01347a.htm (accessed March 1, 2018); John Bolen, "The Wax Candle in the Liturgy," Trinity Communications, https:// www.catholicculture.org/culture/library/view. cfm?recnum=6206 (accessed March 1, 2018).

3. Giovanni Francesco Gemelli Careri, $A$ Voyage to the Philippines (Manila: Filipiniana Book Guild, 1963), 126-27.

4. See, for example Ministerio de Educación, Cultura y Deporte, Archivo General de Indias, Filipinas 203, F.76v, F.437v, F. 727r (in González Research Report to La Follette, June 2015, 6-7); Ministerio de Educación, Cultura y Deporte, Archivo General de Indias, Filipinas 211, IM 157 (in González Research Report to La Follette, March 2017, 29); and Ministerio de Educación, Cultura y Deporte, Archivo General de Indias, Filipinas, 127, N.19, IM 61-62 (in González Research Report to La Follette, October 2016, 13).

5. Russell Skowronek, "Cinnamon, Ceramics and Silks: Tracking the Manila Galleon Trade in the Creation of the World Economy," in Chunming Wu, ed., Early Navigation in the 
Asia-Pacific Region: A Maritime Archaeological Perspective (Singapore: Springer Science + Business Media, 2016), 62.

6. Ibid., 62-66. For a thorough analysis of Chinese porcelains found at Netarts, see Herbert K. Beals and Harvey Steele, Chinese Porcleains from the Site 35-T1-1, Netarts Sand Spit, Tillamook County, Oregon (Eugene: University of Oregon Anthropological Papers no. 23, 1981).

7. Cook, Flood Tide of Empire: Spain and the Pacific Northwest, 1543-1819, 34-40; Fish, The Manila-Acapulco Galleons: The Treasure Ships of the Pacific, 506-507.

8. Ministerio de Educación, Cultura y Deporte, Archivo General de Indias, Filipinas, 204, N.1, F.556R-557V (in González Research Report to La Follette, October 2016, 11-12).

9. Ministerio de Educación, Cultura y Deporte, Archivo General de Indias, Filipinas, 127, N.19, IM. 60-70 (In González Research Report to La Follette, March 2017, 17-18).

10. Ministerio de Educación, Cultura y Deporte, Archivo General de Indias, Filipinas, 187, N.17, IM. 32-33, 37 (in González Research Report to La Follette, March 2017, 27).

11. Ministerio de Educación, Cultura y Deporte, Archivo General de Indias, Filipinas, 129, N.39BIS (in González Research Report to La Follette, October 2016, 14).

12. Ministerio de Educación, Cultura y Deporte, Archivo General de Indias, Filipinas, 127 , N.19, IM. 61-62, 68, 72-73 (in González Research Report to La Follette, October 2016, 12-13).

13. Ministerio de Educación, Cultura y Deporte, Archivo General de Indias, Filipinas, 129, N.1 (in González Research Report to La Follette, March 2017, 24).

14. The digital appendix of the San Francisco Xavier's cargo list is available for download on the Oregon Historical Society website, http://ohs.org/beeswaxwreck or via JSTOR with the digital version of the Summer 2018 issue of the Oregon Historical Quarterly.

15. Schurz, The Manila Galleon, 159-64; Arturo Giraldez, The Age of Trade: The Manila Galleons and the Dawn of the Global Economy. (Lanham, Md.: Rowman \& Littlefield, 2015), 154-57.

16. Schurz, The Manila Galleon, 163-64.
17. Esther González, email to La Follette, November 27-28, 2016; Schurz, The Manila Galleon, 179-80.

18. Ministerio de Educación, Cultura y Deporte, Archivo General de Indias, Filipinas, 214, N.1, F.54R-154V (in González Research Report to La Follette, November 2015, 7-59).

19. Cook, Flood Tide of Empire, 34-35. Quoting "Compte du produit de la cargison du Galion appellee le St. francois Xavier du port doe 1000. Thonneaux venue de Manille a Acapulco au Commencement de mois de Janvier 1707," in Jean de Monségur, "Nouveau Mémoires touchant le Mexique ou la Nouvelle Espagne," 1707-08, FrBN (Ms. Fr. 24228), 339-41. See also E.W. Geisecke, Beeswax, Teak and Castaways: Searching for Oregon's Lost Protohistoric Asian Ship, (Nehalem, OR: Nehalem Valley Historical Society, 2007) for correspondence with Cook over cargo and other details of the San Francisco Xavier of 1705 .

20. Ministerio de Educación, Cultura y Deporte, Archivo General de Indias, Filipinas, 119, N.33, Filipinas, 119, N.34, IM. 2; and Filipinas 129, N.5, IM. 5 (in González Research Report to La Follette, March 2017, 23-25).

21. Especially frustrating was the fact that the archives do contain the official directive for removal and listing of cargo (due to damage and need to replace items) after the Santo Cristo's return to the Philippines in 1692, but the actual itemized cargo list has been lost.

22. Ministerio de Educación, Cultura y Deporte, Archivo General de Indias, Escribania, 442D. Book 1 and Book 2, F. 88R-89R, F.89V (in González Research Report to La Follette, March 2016, 1).

23. Ministerio de Educación, Cultura y Deporte, Archivo General de Indias Escribania, 442D, Book 2, F.93R, F.95R, F.100V-103V, F.105R -114R, F.118V (in González Research Report to La Follette, March 2016, 3).

24. Ministerio de Educación, Cultura y Deporte, Archivo General de Indias, Filipinas, 28, N.147, F.1261-1267 (in González Research Report to La Follette, March 2016, 19).

25. Giraldez, The Age of Trade, 152-53; Schurz, The Manila Galleon, 32. In addition to smuggling on board the galleons, there was 
a lively smuggling trade of Chinese silks between Mexico and Peru, since the profits to be made in Lima ran to 200 percent or higher in the seventeenth and early eighteenth centuries.

26. Schurz, The Manila Galleon, 32; Giraldez, The Age of Trade: The Manila Galleons and the Dawn of the Global Economy, 148.

27. Schurz, The Manila Galleon, 182-83.

28. Don Marshall, Oregon Shipwrecks. (Portland, Ore.: Binford \& Mort Publishing, 1984), 182.

29. Ministerio de Educación, Cultura y Deporte, Archivo General de Indias, Filipinas, 214, N.1, F.58R, F.103R, F.110V, F.133R (in González Research Report to La Follette, November 2015, 2-6).

30. Albaro Alonso Barba, The Art of Metals in which is declared the manner Of Their Generation And The Concomitants of them: In Two Books, written in Spanish by Albaro Alonso Barba, Master of Art, Curate of St. Bernards Parish in the Imperial City of Potosi, in the Kingdom of Peru in the West-Indies, in the Year, 1640, translated in the year 1669, by the R.H. Edward Earl of Sandwich (London: Printed for S. Mearne, Stationer to the Kings Most Excellent Majesty, 1674), 138-41.

31. Kris Lane, "Potosí Mines," part of Latin American History, Oxford Research Encyclopedias, Online Publication, May 2015, http://latinamericanhistory.oxfordre.com/view/10.1093/ acrefore/9780199366439.001.0001/acrefore9780199366439-e-2 (accessed March 6, 2018).

32. Esther González Pérez, "The Trade in Chinese Mercury via the Philippines in the 16th and 17th Centuries," May 2016, translated Tragora Traducciones August 2016 (unpublished paper in La Follette's possession), 1-4.

33. Ibid., 4. For a comprehensive study of Spanish Andean silver mining, see Nicholas A. Robins, Mercury Mining and Empire: The Human and Ecological Cost of Colonial Silver Mining in the Andes (Bloomington: Indiana University Press, 2011).

34. González, "The Trade in Chinese Mercury via the Philippines in the 16th and 17th Centuries," 3-5.

35. Ibid., 13-15.

36. Guillermina del Valle Pavón, “Bases del poder de los mercaderes de plata de la ciudad de México. Redes, control del Consulado y de la Casa de Moneda a fines del siglo XVII," Anuario de estudios americanos, 68:2 (2011): 583 (in González Mercury References List to La Follette, July 2016, 1); González, "The Trade in Chinese Mercury via the Philippines in the 16th and 17th Centuries," 14.

37. Esther González email to La Follette, July 22, 2016 (in González-La Follette emails regarding Mercury Shipment in Manila Trade, July-October 2016, 1-3); "The Quicksilver Galleons," DiscoverSea Shipwreck Museum, http://www.discoversea.com/The-QuicksilverGalleons.html (accessed June 22, 2017); ); Mendel Peterson, "Graveyard of the Quicksilver Galleons," National Geographic, December 1979, 867.

38. Esther González email to La Follette, October 4, 2016 (in González-La Follette Emails regarding Mercury Shipment in Manila Trade, July-October 2016, 2).

39. Ministerio de Educación, Cultura y Deporte, Archivo General de Indias, Filipinas, 15, R.1, N.43, IM. 3 (in González Mercury References on SCB, July 2016, 1).

40. Mendel Peterson, "Graveyard of the Quicksilver Galleons," National Geographic, December 1979, 851-69.

41. Chad R. Hammerschmidt, "Mercury and Carbon Dioxide Emissions: Uncoupling a Toxic Relationship," Environmental Toxicology and Chemistry, 30:12 (December 2011): 2640.

42. Carl Lamborg, et. al., "Mercury in the Anthropocene Ocean," Oceanography 27:1 (March 2014): 76-87.

43. Genevieve Wanucha, "Inside Quicksilver's Toxic Transformation," March 7, 2013, available online at http://oceans.mit.edu/news/ featured-stories/quicksilver-consequences (accessed January 2017), 1-2.

44. Lamborg et.al., "Mercury in the Anthropocene Ocean," 78-81; and United National Environment Programme, Global Mercury Assessment 2013: Sources, Emissions, Releases and Environmental Transport. (Geneva, Switzerland: United Nations Environment Programme, 2013), 3-7, 26-29.

45. Wanucha, "Inside Quicksilver's Toxic Transformation," $1-2$. 


\section{COMPLETE CARGO LIST FOR THE 1701 SAN FRANCISCO XAVIER}

Shippers' marks for each consignment item are pictured to the left of the list item.

\begin{tabular}{|c|c|c|c|}
\hline $\begin{array}{l}\text { SHIPPER'S } \\
\text { MARK }\end{array}$ & SHIPPER & MERCHANDISE & SOURCE \\
\hline & $\begin{array}{l}\text { Captain Diego } \\
\text { Vivien }\end{array}$ & $\begin{array}{c}\text { white cotton clothes, blankets from China, satins } \\
\text {, silks, towels, petticoats, fabrics, chocolate } \\
\text { bowls }\end{array}$ & Filipinas,214,N.1,F.54r \\
\hline & $\begin{array}{c}\text { General } \\
\text { Bernardo de } \\
\text { Endaya }\end{array}$ & fabrics, blankets from China & Filipinas,214,N.1,F.56r \\
\hline & $\begin{array}{l}\text { Captain Agustín } \\
\text { Crespo }\end{array}$ & $\begin{array}{l}\text { satins, silks, blankets from China, white cotton } \\
\text { clothes, blankets from China }\end{array}$ & Filipinas,214,N.1,F.56v \\
\hline & $\begin{array}{c}\text { Sergeant Major } \\
\text { Fernando } \\
\text { Iglesias }\end{array}$ & towels, blankets from China & Filipinas,214,N.1,F.57v \\
\hline & $\begin{array}{l}\text { Captain Fran- } \\
\text { cisco Manuel } \\
\text { de Yrrasagoria }\end{array}$ & white cotton clothes, fabrics & Filipinas, 214,N.1,F.58r \\
\hline & $\begin{array}{l}\text { Captain Fran- } \\
\text { cisco Manuel } \\
\text { de Yrrasagoria }\end{array}$ & white cotton clothes, fabrics & Filipinas, 214,N.1,F.58r \\
\hline & $\begin{array}{l}\text { Captain Fran- } \\
\text { cisco Manuel } \\
\text { de Yrrasagoria }\end{array}$ & $\begin{array}{c}18 \text { "medias marquetas " of bee wax, third mark, } \\
\text { number } 1 \text { to } 18 \text {, each of them weighing } 3 \text { arrobas } \\
(621 \mathrm{~kg})\end{array}$ & Filipinas,214,N.1,F.58r \\
\hline
\end{tabular}

THIS DETAILED CARGO MANIFEST shows all properly registered cargo, including the names of the shippers and their identification marks, carried on the San Francisco Xavier galleon voyage from Manila to Acapulco in 1701. The manifest was deposited in the Archivo General de Indias (General Archive of the Indies) in Seville, Spain. This is the official archive for documents of Spain's empire in the Philippines and the Americas. Any smuggled cargo, which was commonplace in the Manila trade, is not listed on the manifest. 


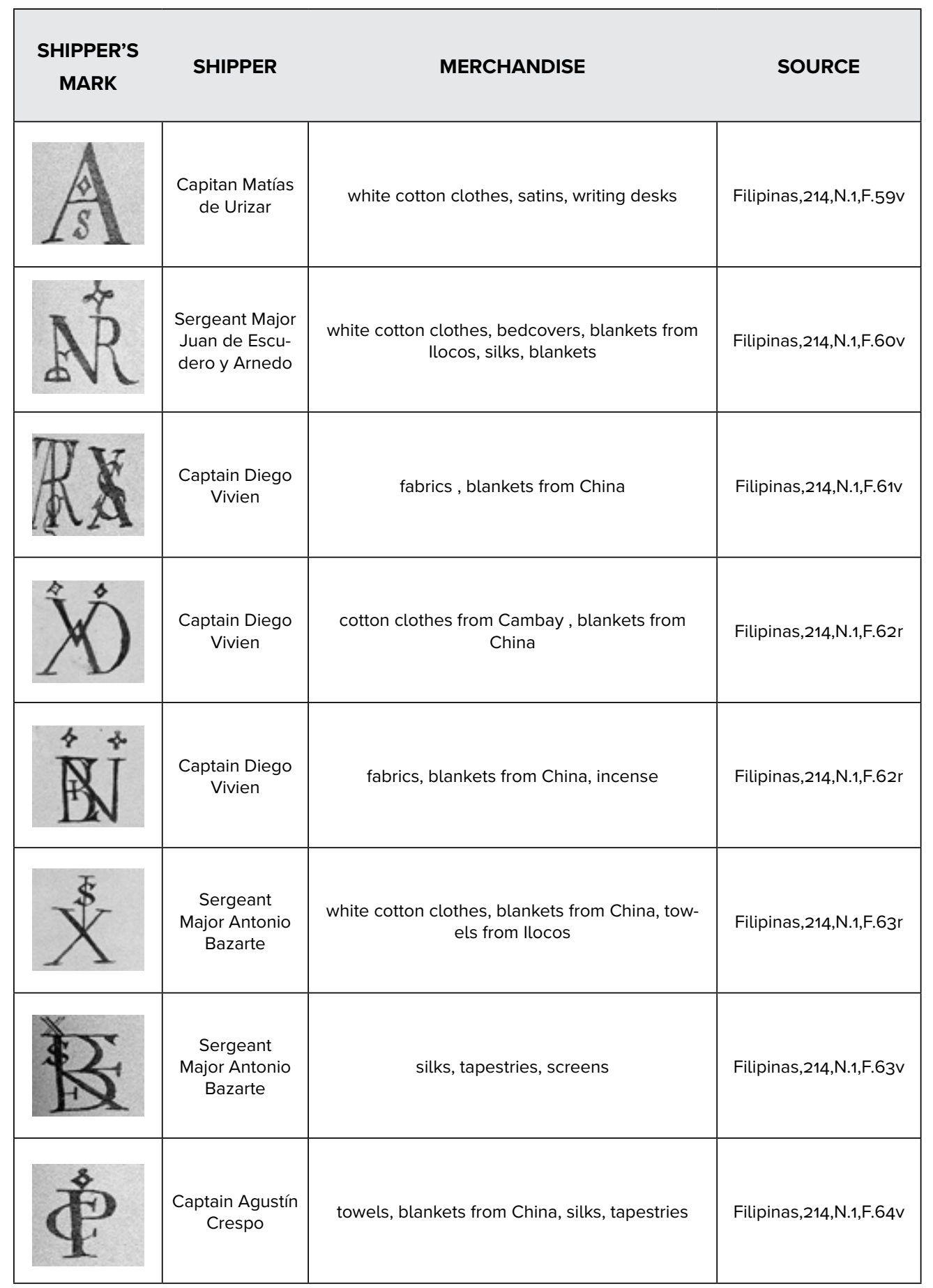




\begin{tabular}{|c|c|c|c|}
\hline $\begin{array}{l}\text { SHIPPER'S } \\
\text { MARK }\end{array}$ & SHIPPER & MERCHANDISE & SOURCE \\
\hline & $\begin{array}{l}\text { Captain Agustín } \\
\text { Crespo }\end{array}$ & white cotton clothes, blankets from China & Filipinas,214,N.1,F.65V \\
\hline & $\begin{array}{l}\text { Captain Agustín } \\
\text { Crespo }\end{array}$ & $\begin{array}{l}\text { silks, fans, blankets from China, white cotton } \\
\text { clothes }\end{array}$ & Filipinas,214,N.1,F.66r \\
\hline & $\begin{array}{c}\text { General José } \\
\text { de Robles } \\
\text { Cortés }\end{array}$ & silks, damasks, towels & Filipinas,214,N.1,F.67r \\
\hline & $\begin{array}{c}\text { General } \\
\text { Marcos } \\
\text { Quintero } \\
\text { Ramos }\end{array}$ & white cotton clothes, blankets from China & Filipinas, 214,N.1,F.67v \\
\hline & $\begin{array}{c}\text { General } \\
\text { Marcos } \\
\text { Quintero } \\
\text { Ramos }\end{array}$ & $\begin{array}{l}\text { petticoats, blankets from China, cotton clothes } \\
\text { from Cambay, blankets }\end{array}$ & Filipinas, 214,N.1,F.68r \\
\hline & $\begin{array}{c}\text { General } \\
\text { Marcos } \\
\text { Quintero } \\
\text { Ramos }\end{array}$ & towels, blankets from China & Filipinas, 214,N.1,F.68r \\
\hline & $\begin{array}{c}\text { General } \\
\text { Marcos } \\
\text { Quintero } \\
\text { Ramos }\end{array}$ & white cotton clothes, blankets & Filipinas, 214,N.1,F.68v \\
\hline$y$ & $\begin{array}{c}\text { General } \\
\text { Marcos } \\
\text { Quintero } \\
\text { Ramos }\end{array}$ & white cotton clothes, blankets & Filipinas, 214,N.1,F.68v \\
\hline
\end{tabular}




\begin{tabular}{|c|c|c|c|}
\hline $\begin{array}{c}\text { SHIPPER'S } \\
\text { MARK }\end{array}$ & SHIPPER & MERCHANDISE & SOURCE \\
\hline & $\begin{array}{c}\text { General } \\
\text { Marcos } \\
\text { Quintero } \\
\text { Ramos }\end{array}$ & white cotton clothes, blankets & Filipinas,214,N.1,F.68v \\
\hline & $\begin{array}{c}\text { General } \\
\text { Marcos } \\
\text { Quintero } \\
\text { Ramos }\end{array}$ & white cotton clothes, blankets & Filipinas,214,N.1,F.69r \\
\hline & $\begin{array}{c}\text { General } \\
\text { Marcos } \\
\text { Quintero } \\
\text { Ramos }\end{array}$ & white cotton clothes, blankets & Filipinas,214,N.1,F.69r \\
\hline & $\begin{array}{c}\text { General } \\
\text { Marcos } \\
\text { Quintero } \\
\text { Ramos }\end{array}$ & white cotton clothes, blankets & Filipinas,214,N.1,F.69r \\
\hline & $\begin{array}{c}\text { General } \\
\text { Marcos } \\
\text { Quintero } \\
\text { Ramos }\end{array}$ & white cotton clothes, blankets & Filipinas,214,N.1,F.69v \\
\hline & $\begin{array}{c}\text { General } \\
\text { Marcos } \\
\text { Quintero } \\
\text { Ramos }\end{array}$ & white cotton clothes, blankets & Filipinas,214,N.1,F.69v \\
\hline & $\begin{array}{c}\text { General } \\
\text { Marcos } \\
\text { Quintero } \\
\text { Ramos }\end{array}$ & white cotton clothes, blankets & Filipinas,214,N.1,F.69v \\
\hline & $\begin{array}{c}\text { General } \\
\text { Marcos } \\
\text { Quintero } \\
\text { Ramos }\end{array}$ & white cotton clothes, blankets & Filipinas,214,N.1,F.69v \\
\hline
\end{tabular}




\begin{tabular}{|c|c|c|c|}
\hline $\begin{array}{c}\text { SHIPPER'S } \\
\text { MARK }\end{array}$ & SHIPPER & MERCHANDISE & SOURCE \\
\hline & $\begin{array}{l}\text { General } \\
\text { Marcos } \\
\text { Quintero } \\
\text { Ramos }\end{array}$ & white cotton clothes, blankets & Filipinas,214,N.1,F.70r \\
\hline & $\begin{array}{l}\text { Juan de Eche- } \\
\text { varria }\end{array}$ & $\begin{array}{c}\text { white cotton clothes, blankets from China, silks, } \\
\text { tapestries, towels, faience, chocolate cups, } \\
\text { trunks, writing desks, screens }\end{array}$ & Filipinas,214,N.1,F.70rv \\
\hline & Ana Rayo & $\begin{array}{c}\text { cotton clothes from Cambay, blankets from } \\
\text { China }\end{array}$ & Filipinas, 214,N.1,F.72r \\
\hline & $\begin{array}{l}\text { General Fran- } \\
\text { cisco Archoca }\end{array}$ & $\begin{array}{c}\text { cotton clothes from Cambay, blankets from } \\
\text { China }\end{array}$ & Filipinas, 214,N.1,F.72v \\
\hline & $\begin{array}{l}\text { Captain Martín } \\
\text { de Yriarte }\end{array}$ & $\begin{array}{c}\text { white cotton clothes, blankets from China, a } \\
\text { block of wax, weighing } 3 \text { arrobas }(34,5 \mathrm{~kg}) \text {, silks, } \\
\text { damasks }\end{array}$ & Filipinas, 214,N.1,F.73r \\
\hline & $\begin{array}{l}\text { Captain } \\
\text { Antonio Ruiz } \\
\text { Moreno }\end{array}$ & $\begin{array}{l}\text { white cotton clothes, blankets from China, satins, } \\
\text { silks, blankets }\end{array}$ & Filipinas, 214,N.1,F.74r \\
\hline & $\begin{array}{l}\text { Captain Fran- } \\
\text { cisco Afán de } \\
\text { Ribera }\end{array}$ & white cotton clothes, blankets from China & Filipinas, 214,N.1,F.75V \\
\hline & $\begin{array}{c}\text { Captain Juan } \\
\text { Esteban de } \\
\text { Encinas }\end{array}$ & silks, fans, blankets, satins, blankets from China & Filipinas, 214,N.1,F.76v \\
\hline
\end{tabular}




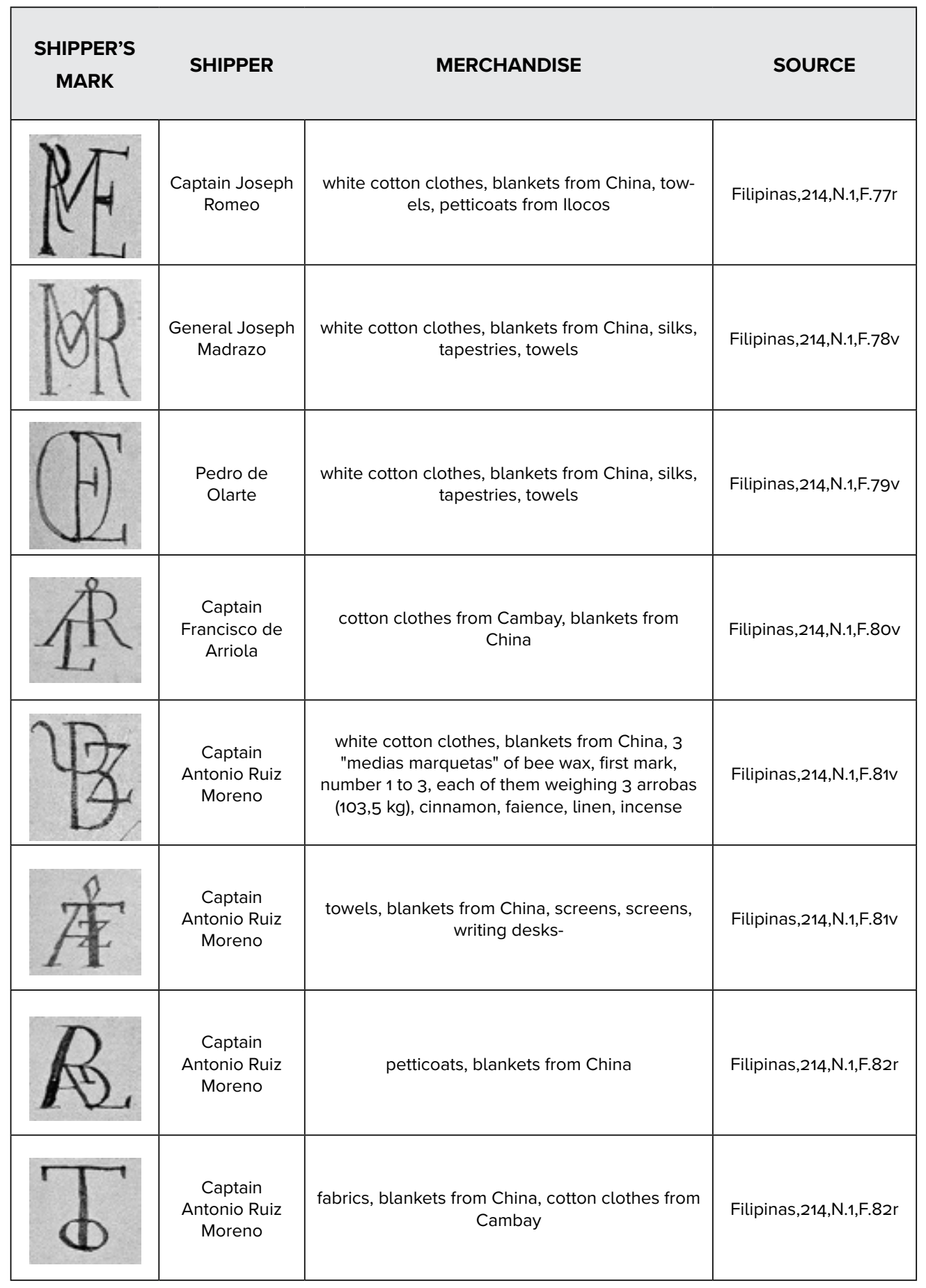




\begin{tabular}{|c|c|c|c|}
\hline $\begin{array}{c}\text { SHIPPER'S } \\
\text { MARK }\end{array}$ & SHIPPER & MERCHANDISE & SOURCE \\
\hline & $\begin{array}{c}\text { Captain } \\
\text { Antonio Ruiz } \\
\text { Moreno }\end{array}$ & towels, blankets from China & Filipinas,214,N.1,F.82v \\
\hline & $\begin{array}{c}\text { Captain } \\
\text { Antonio Ruiz } \\
\text { Moreno }\end{array}$ & $\begin{array}{l}\text { cotton clothes from Cambay, blankets from } \\
\text { China }\end{array}$ & Filipinas,214,N.1,F.82v \\
\hline & $\begin{array}{l}\text { Captain } \\
\text { Fernando de } \\
\text { Limos }\end{array}$ & $\begin{array}{l}\text { white cotton clothes, blankets from China, writ- } \\
\text { ing desks, chocolate bowls }\end{array}$ & Filipinas,214,N.1,F.84V \\
\hline & $\begin{array}{c}\text { Captain } \\
\text { Fernando de } \\
\text { Limos }\end{array}$ & $\begin{array}{l}\text { cotton clothes from Cambay, blankets from } \\
\text { China, silks, tapestries, towels }\end{array}$ & Filipinas,214,N.1,F.85r \\
\hline & $\begin{array}{l}\text { Captain } \\
\text { Fernando de } \\
\text { Limos }\end{array}$ & silks, fans, blankets from China & Filipinas,214,N.1,F.85V \\
\hline & $\begin{array}{l}\text { Captain } \\
\text { Fernando de } \\
\text { Limos }\end{array}$ & fabrics, blankets from China, faience & Filipinas,214,N.1,F.85V \\
\hline & $\begin{array}{l}\text { Captain Diego } \\
\text { Zúñiga }\end{array}$ & white cotton clothes, blankets from China & Filipinas,214,N.1,F.86r \\
\hline & $\begin{array}{l}\text { Captain Juan } \\
\text { Ignacio } \\
\text { Castellanos }\end{array}$ & $\begin{array}{l}\text { cotton clothes from Cambay, blankets from } \\
\text { China }\end{array}$ & Filipinas,214,N.1,F.87r \\
\hline
\end{tabular}




\begin{tabular}{|c|c|c|c|}
\hline $\begin{array}{l}\text { SHIPPER'S } \\
\text { MARK }\end{array}$ & SHIPPER & MERCHANDISE & SOURCE \\
\hline & $\begin{array}{l}\text { Captain Juan } \\
\quad \text { Ignacio } \\
\text { Castellanos }\end{array}$ & $\begin{array}{c}\text { white cotton clothes, blankets from China, cotton } \\
\text { clothes, damasks, cotton clothes from Cambay, } \\
\text { satins, faience }\end{array}$ & Filipinas, 214,N.1,F.87r \\
\hline & $\begin{array}{l}\text { Second } \\
\text { Liutenant Blas } \\
\text { de Espinosa }\end{array}$ & petticoats, blankets from China & Filipinas,214,N.1,F.88v \\
\hline & $\begin{array}{l}\text { Second } \\
\text { Liutenant } \\
\text { Joseph } \\
\text { Hernández }\end{array}$ & bowls & Filipinas,214,N.1,F.89r \\
\hline & $\begin{array}{l}\text { Second } \\
\text { Liutenant } \\
\text { Joseph } \\
\text { Hernández }\end{array}$ & bowls & Filipinas,214,N.1,F.89v \\
\hline & $\begin{array}{l}\text { Captain Diego } \\
\text { de Zelada }\end{array}$ & $\begin{array}{l}\text { white cotton clothes, blankets from China, silks, } \\
\text { tapestries, towels }\end{array}$ & Filipinas,214,N.1,F.9or \\
\hline & $\begin{array}{l}\text { Sergeant Major } \\
\text { Juan Antonio } \\
\text { de Ybarra }\end{array}$ & $\begin{array}{l}\text { fabrics, blankets from China, } 10 \text { "medias marqu- } \\
\text { etas" of bee wax, first mark, number } 1 \text { to } 10 \text {, each } \\
\text { of them weighing } 3 \text { arrobas ( } 345 \mathrm{~kg} \text { )" }\end{array}$ & Filipinas, 214,N.1,F.91r \\
\hline & $\begin{array}{l}\text { Sergeant Major } \\
\text { Juan Antonio } \\
\text { de Ybarra }\end{array}$ & $\begin{array}{c}\text { cotton clothes from Cambay, blankets from } \\
\text { China }\end{array}$ & Filipinas, 214,N.1,F.91r \\
\hline & $\begin{array}{l}\text { Sergeant Major } \\
\text { Juan Antonio } \\
\text { de Ybarra }\end{array}$ & silks, tapestries, towels, blankets & Filipinas,214,N.1,F.91V \\
\hline
\end{tabular}




\begin{tabular}{|c|c|c|c|}
\hline SHIPPER'S & SHIPPER & MERCHANDISE & SOURCE \\
\hline & $\begin{array}{l}\text { General Joseph } \\
\text { de Robles }\end{array}$ & white cotton clothes, blankets from China & Filipinas,214,N.1,F.92v \\
\hline & $\begin{array}{l}\text { Captain Joseph } \\
\text { de Sarralde }\end{array}$ & towels, blankets from China, silks, fans, blankets & Filipinas,214,N.1,F.93r \\
\hline & María de Castro & $\begin{array}{c}\text { cotton clothes, damasks, cotton clothes from } \\
\text { Cambay, satins }\end{array}$ & Filipinas,214,N.1,F.94V \\
\hline & $\begin{array}{c}\text { Sergeant Major } \\
\text { Juan de Escaño } \\
\text { y Córdoba }\end{array}$ & $\begin{array}{l}\text { petticoats, blankets from China, silks, damasks, } \\
\text { towels }\end{array}$ & Filipinas,214,N.1,F.95V \\
\hline & $\begin{array}{l}\text { Captain Agustin } \\
\text { de Torralva }\end{array}$ & $\begin{array}{l}\text { cotton clothes, damasks, cotton clothes from } \\
\text { Cambay, satins, silks, blankets }\end{array}$ & Filipinas, 214,N.1,F.96v \\
\hline & $\begin{array}{l}\text { Clara de } \\
\text { Ampuero }\end{array}$ & $\begin{array}{l}\text { cotton clothes, damasks, cotton clothes from } \\
\text { Cambay, satins }\end{array}$ & Filipinas,214,N.1,F.97v \\
\hline & $\begin{array}{l}\text { Assistant Simón } \\
\text { Silvestre }\end{array}$ & $\begin{array}{l}\text { cotton clothes, damasks, cotton clothes from } \\
\text { Cambay, satins }\end{array}$ & Filipinas,214,N.1,F.98r \\
\hline & $\begin{array}{l}\text { Sergeant Major } \\
\text { Sebastián de } \\
\text { Oya y Troncoso }\end{array}$ & A box with presents & Filipinas,214,N.1,F.99r \\
\hline
\end{tabular}




\begin{tabular}{|c|c|c|c|}
\hline $\begin{array}{l}\text { SHIPPER'S } \\
\text { MARK }\end{array}$ & SHIPPER & MERCHANDISE & SOURCE \\
\hline & $\begin{array}{l}\text { Fray Domingo } \\
\text { de Escalera } \\
\text { (Dominico) }\end{array}$ & liturgical and religious objects, working clothing & Filipinas,214,N.1,F.99v \\
\hline & $\begin{array}{l}\text { General Tomás } \\
\text { Antonio Mar- } \\
\text { tínez }\end{array}$ & $\begin{array}{l}\text { white cotton clothes, blankets from China, silks, } \\
\text { faience bowls, faience horses, faience lions }\end{array}$ & Filipinas,214,N.1,F.100V \\
\hline & $\begin{array}{l}\text { Captain Juan } \\
\text { García Infanzón }\end{array}$ & $\begin{array}{l}\text { fabrics, blankets from China, silks, tapestries, } \\
\text { towels }\end{array}$ & Filipinas,214,N.1,F.101V \\
\hline & $\begin{array}{l}\text { Sergeant Major } \\
\text { Francisco de } \\
\text { Maya y Torres }\end{array}$ & $\begin{array}{l}\text { white cotton clothes, blankets from China, silks, } \\
\text { blankets, fabrics, writing desks, one screen }\end{array}$ & Filipinas,214,N.1,F.103r \\
\hline & $\begin{array}{l}\text { Sergeant Major } \\
\text { Francisco de } \\
\text { Maya y Torres }\end{array}$ & $\begin{array}{l}\text { white cotton clothes, blankets from China, silks, } \\
\text { fans, blankets, one screen, writing desks }\end{array}$ & Filipinas,214,N.1,F.104v \\
\hline & $\begin{array}{l}\text { Sergeant Major } \\
\text { Francisco de } \\
\text { Maya y Torres }\end{array}$ & $\begin{array}{l}\text { cotton clothes from Cambay, blankets from } \\
\text { China, silks, tapestries, towels, blankets from } \\
\text { China }\end{array}$ & Filipinas,214,N.1,F.105r \\
\hline & $\begin{array}{l}\text { Sergeant Major } \\
\text { Francisco de } \\
\text { Maya y Torres }\end{array}$ & $\begin{array}{l}\text { petticoats, blankets from China, silks, damasks, } \\
\text { towels }\end{array}$ & Filipinas,214,N.1,F.105V \\
\hline & $\begin{array}{l}\text { Sergeant Major } \\
\text { Joseph de } \\
\text { Aguirre }\end{array}$ & $\begin{array}{c}\text { fabrics, blankets from China, cinnamon from } \\
\text { Burney, incense, pepper }\end{array}$ & Filipinas, 214,N.1,F.106v \\
\hline
\end{tabular}




\begin{tabular}{|c|c|c|c|}
\hline $\begin{array}{l}\text { SHIPPER'S } \\
\text { MARK }\end{array}$ & SHIPPER & MERCHANDISE & SOURCE \\
\hline & $\begin{array}{c}\text { Sergeant Major } \\
\text { Joseph de } \\
\text { Aguirre }\end{array}$ & silks, tapestries, towels, blankets from China & Filipinas,214,N.1,F.107r \\
\hline & $\begin{array}{l}\text { Sergeant Major } \\
\text { Joseph de } \\
\text { Aguirre }\end{array}$ & silks, damasks, towels & Filipinas,214,N.1,F.107r \\
\hline & $\begin{array}{l}\text { Sergeant Major } \\
\text { Joseph de } \\
\text { Aguirre }\end{array}$ & $\begin{array}{l}\text { towels, blankets from China, silks, fans, blankets, } \\
\text { writing desks, faience, cinnamon from Burney }\end{array}$ & Filipinas,214,N.1,F.107r \\
\hline & $\begin{array}{c}\text { Sergeant Major } \\
\text { Joseph de } \\
\text { Aguirre }\end{array}$ & fabrics, blankets from China & Filipinas,214,N.1,F.107V \\
\hline & $\begin{array}{l}\text { Sergeant Major } \\
\text { Joseph de } \\
\text { Aguirre }\end{array}$ & $\begin{array}{c}\text { cotton clothes from Cambay, blankets from } \\
\text { China }\end{array}$ & Filipinas, 214,N.1,F.108r \\
\hline & $\begin{array}{l}\text { Sergeant Major } \\
\text { Joseph de } \\
\text { Aguirre }\end{array}$ & $\begin{array}{c}14 \text { "medias marquetas" of bee wax, seventh } \\
\text { mark, number } 1 \text { to } 14 \text {, each of them weighing } 3 \\
\text { arrobas ( } 483 \mathrm{~kg})\end{array}$ & Filipinas,214,N.1,F.108v \\
\hline & $\begin{array}{l}\text { Sergeant Major } \\
\text { Joseph de } \\
\text { Aguirre }\end{array}$ & writing desk, faience, screens, one cot & Filipinas,214,N.1,F.109r \\
\hline & $\begin{array}{l}\text { Sergeant Major } \\
\text { Joseph de } \\
\text { Aguirre }\end{array}$ & writing desks, writing desks, trunks, screens & Filipinas,214,N.1,F.109r \\
\hline
\end{tabular}




\begin{tabular}{|c|c|c|c|}
\hline $\begin{array}{l}\text { SHIPPER'S } \\
\text { MARK }\end{array}$ & SHIPPER & MERCHANDISE & SOURCE \\
\hline & $\begin{array}{l}\text { Sergeant Major } \\
\text { Joseph de } \\
\text { Aguirre }\end{array}$ & silks, tapestries, towels, blankets from China & Filipinas,214,N.1,F.109v \\
\hline & $\begin{array}{l}\text { Captain Joseph } \\
\text { de Ozcorta }\end{array}$ & petticoats, blankets from China & Filipinas,214,N.1,F.110v \\
\hline & $\begin{array}{l}\text { Captain Joseph } \\
\text { de Ozcorta }\end{array}$ & $\begin{array}{l}\text { cotton clothes from Cambay, blankets from } \\
\text { China, silks, tapestries, towels }\end{array}$ & Filipinas,214,N.1,F.110V \\
\hline & $\begin{array}{l}\text { Captain Joseph } \\
\text { de Ozcorta }\end{array}$ & white cotton clothes, blankets from China & Filipinas,214,N.1,F.110V \\
\hline & $\begin{array}{l}\text { Captain Joseph } \\
\text { de Ozcorta }\end{array}$ & fabrics, blankets from China & Filipinas,214,N.1,F.110V \\
\hline & $\begin{array}{l}\text { Captain Andrés } \\
\text { de Pividal }\end{array}$ & silks, damasks, towels & Filipinas,214,N.1,F.111r \\
\hline & Joseph Marzán & faience & Filipinas,214,N.1,F.111V \\
\hline & $\begin{array}{c}\text { María Pérez } \\
\text { Espinosa de los } \\
\text { Monteros }\end{array}$ & $\begin{array}{l}\text { cotton clothes from Cambay, blankets from } \\
\text { China }\end{array}$ & Filipinas,214,N.1,F.112r \\
\hline
\end{tabular}




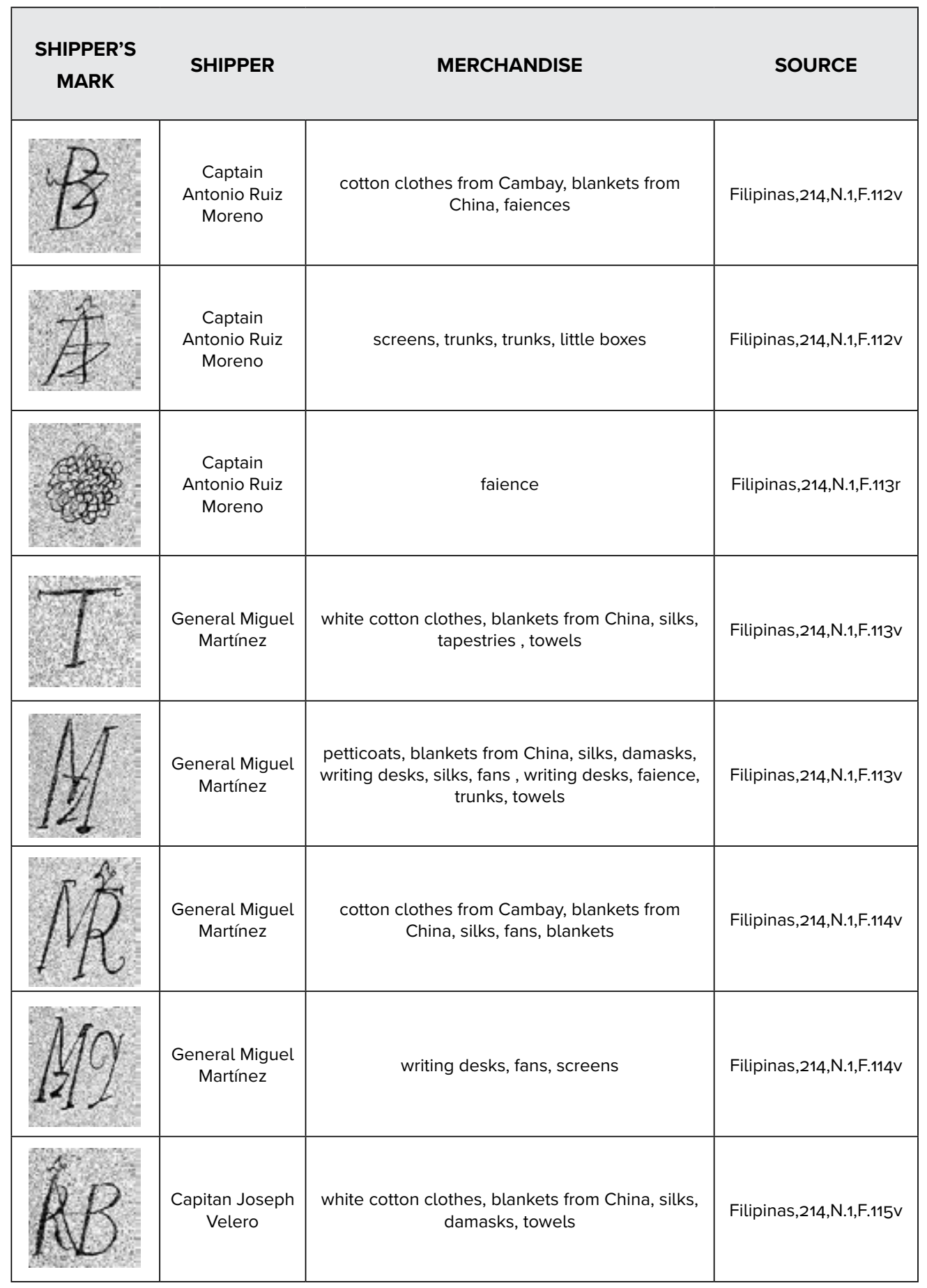




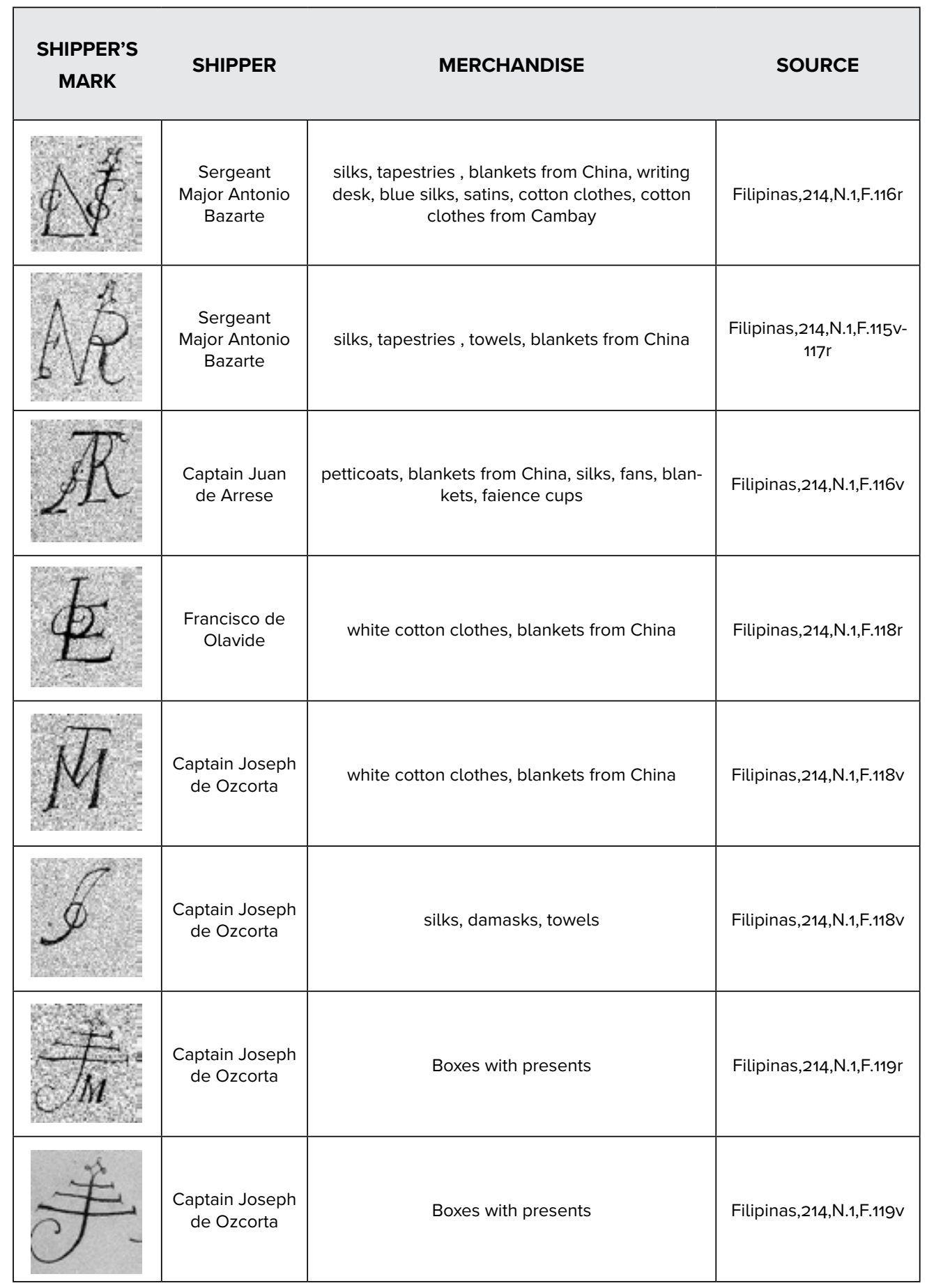




\begin{tabular}{|c|c|c|c|}
\hline $\begin{array}{c}\text { SHIPPER'S } \\
\text { MARK }\end{array}$ & SHIPPER & MERCHANDISE & SOURCE \\
\hline & $\begin{array}{l}\text { Assistant Pedro } \\
\text { Morales }\end{array}$ & $\begin{array}{l}\text { silks, tapestries, towels, blankets from China, } \\
\text { writing desks, fans, cotton clothes, blue silks }\end{array}$ & Filipinas,214,N.1,F.12or \\
\hline & $\begin{array}{l}\text { Captain Pedro } \\
\text { Luzena }\end{array}$ & $\begin{array}{c}\text { fabrics, silks, tapestries, towels, blankets from } \\
\text { China, satins, blankets, } 12 \text { "medias marquetas" of } \\
\text { bee wax number } 1 \text { to } 12 \text {, next mark, each of them } \\
\text { weighing } 3 \text { arrobas ( } 414 \mathrm{~kg})\end{array}$ & Filipinas,214,N.1,F.121r \\
\hline & $\begin{array}{l}\text { Captain } \\
\text { Fernando de } \\
\text { Angulo }\end{array}$ & $\begin{array}{c}\text { cotton clothes from Cambay, blankets from } \\
\text { China }\end{array}$ & Filipinas,214,N.1,F.122r \\
\hline & $\begin{array}{l}\text { Captain } \\
\text { Fernando de } \\
\text { Angulo }\end{array}$ & $\begin{array}{c}\text { petticoats, blankets from China, silks, damasks, } \\
\text { towels, white cotton clothes, fans, blankets, } \\
\text { fabrics, tapestries }\end{array}$ & Filipinas,214,N.1,F.122r \\
\hline & $\begin{array}{c}\text { Graduate } \\
\text { Joseph de } \\
\text { Arriola }\end{array}$ & $\begin{array}{l}\text { cotton clothes from Cambay, blankets from } \\
\text { China, silks, tapestries }\end{array}$ & Filipinas,214,N.1,F.123V \\
\hline & $\begin{array}{l}\text { Francisco } \\
\text { Munilla }\end{array}$ & $\begin{array}{l}\text { towels, blankets from China, silks, damasks, } \\
\text { faience }\end{array}$ & Filipinas,214,N.1,F.124r \\
\hline No mark & $\begin{array}{l}\text { General Miguel } \\
\text { Martínez }\end{array}$ & bowls & Filipinas,214,N.1,F.124v \\
\hline & $\begin{array}{l}\text { Captain Blas } \\
\text { Bruno }\end{array}$ & $\begin{array}{l}\text { white cotton clothes, blankets from China, silks, } \\
\text { tapestries, towels }\end{array}$ & Filipinas,214,N.1,F.125r \\
\hline
\end{tabular}




\begin{tabular}{|c|c|c|c|}
\hline $\begin{array}{c}\text { SHIPPER'S } \\
\text { MARK }\end{array}$ & SHIPPER & MERCHANDISE & SOURCE \\
\hline & $\begin{array}{c}\text { Captain } \\
\text { Atanasio } \\
\text { Fernández de } \\
\text { Guevara }\end{array}$ & white cotton clothes, blankets from China & Filipinas,214,N.1,F.126v \\
\hline & $\begin{array}{c}\text { Captain } \\
\text { Atanasio } \\
\text { Fernández de } \\
\text { Guevara }\end{array}$ & $\begin{array}{c}\text { silks, tapestries, towels, blankets from China, } \\
\text { blankets }\end{array}$ & Filipinas,214,N.1,F.126v \\
\hline & $\begin{array}{c}\text { Captain } \\
\text { Atanasio } \\
\text { Fernández de } \\
\text { Guevara }\end{array}$ & $\begin{array}{c}\text { petticoats, blankets from China, silks, damasks, } \\
\text { towels, faience }\end{array}$ & Filipinas,214,N.1,F.127r \\
\hline No mark & $\begin{array}{c}\text { Captain } \\
\text { Domingo López }\end{array}$ & $\begin{array}{c}\text { cotton clothes from Cambay, blankets from } \\
\text { China }\end{array}$ & $\begin{array}{l}\text { Filipinas, 214,N.1,F.128v- } \\
\text { 129r }\end{array}$ \\
\hline No mark & $\begin{array}{l}\text { Captain Pedro } \\
\text { Juan Mirado }\end{array}$ & fabrics, blankets from China & Filipinas, 214,N.1,F.129r-v \\
\hline & $\begin{array}{l}\text { Captain Juan } \\
\text { de Arrese }\end{array}$ & $\begin{array}{l}\text { petticoats, blankets from China, silks, damasks, } \\
\text { towels }\end{array}$ & Filipinas,214,N.1,F.130r \\
\hline & $\begin{array}{l}\text { General Juan } \\
\text { de Echeverría }\end{array}$ & $\begin{array}{l}\text { white cotton clothes, blankets from China, silks, } \\
\text { fans }\end{array}$ & Filipinas,214,N.1,F.131r \\
\hline & $\begin{array}{l}\text { General Juan } \\
\text { de Echeverría }\end{array}$ & petticoats, blankets from China & Filipinas,214,N.1,F.131r \\
\hline
\end{tabular}




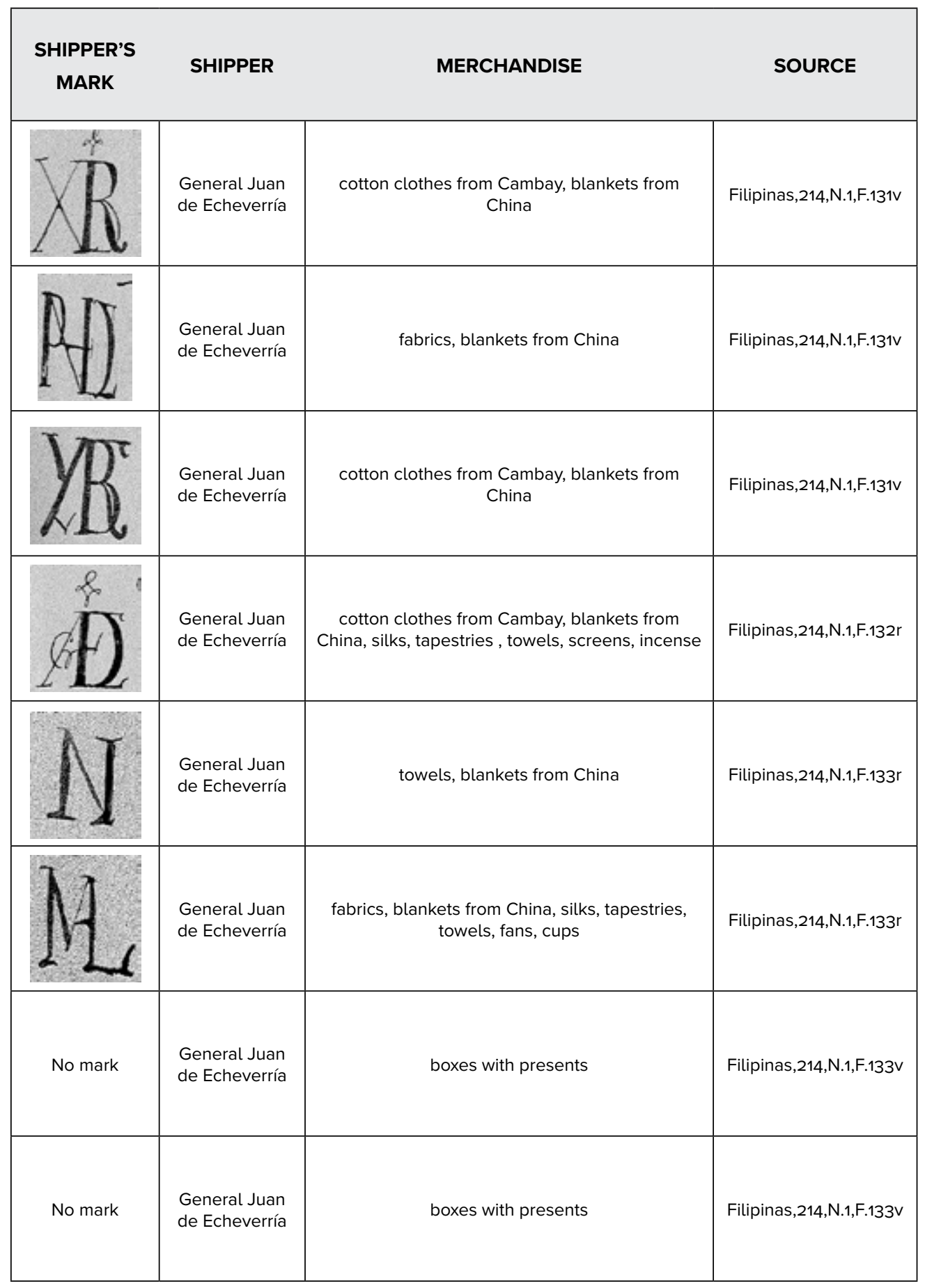




\begin{tabular}{|c|c|c|c|}
\hline $\begin{array}{l}\text { SHIPPER'S } \\
\text { MARK }\end{array}$ & SHIPPER & MERCHANDISE & SOURCE \\
\hline & $\begin{array}{l}\text { Captain } \\
\text { Cristóbal de } \\
\text { Miranda }\end{array}$ & white cotton clothes, blankets from China & Filipinas, 214,N.1,F.133V \\
\hline & $\begin{array}{l}\text { Captain } \\
\text { Cristóbal de } \\
\text { Miranda }\end{array}$ & $\begin{array}{l}\text { towels, blankets from China, cotton clothes, } \\
\text { tapestries }\end{array}$ & Filipinas,214,N.1,F.134r \\
\hline & $\begin{array}{l}\text { Captain Joseph } \\
\text { de Ziriano }\end{array}$ & $\begin{array}{l}\text { white cotton clothes, blankets from China, silks, } \\
\text { fans, cinnamon, incense, faience }\end{array}$ & Filipinas,214,N.1,F.135r \\
\hline & $\begin{array}{l}\text { Captain Pedro } \\
\text { Juan Mirado }\end{array}$ & $\begin{array}{l}\text { cotton clothes from Cambay, silks, tapestries, } \\
\text { towels, blankets from China, incense }\end{array}$ & Filipinas,214,N.1,F.136r \\
\hline & $\begin{array}{l}\text { Sergeant } \\
\text { Major Agustín } \\
\text { Hernández }\end{array}$ & $\begin{array}{l}\text { white cotton clothes, blankets from China, } \\
\text { silks, tapestries, towels, faience, writing desks, } \\
\text { incense, cinnamon }\end{array}$ & Filipinas,214,N.1,F.137r \\
\hline & $\begin{array}{l}\text { Sergeant } \\
\text { Major Agustín } \\
\text { Hernández }\end{array}$ & $\begin{array}{l}\text { silks, damasks, towels, trunks, petticoat, blankets } \\
\text { from China }\end{array}$ & Filipinas,214,N.1,F.138r \\
\hline & $\begin{array}{l}\text { Sergeant } \\
\text { Major Agustín } \\
\text { Hernández }\end{array}$ & $\begin{array}{c}\text { writing desks, trunks, combs, } 6 \text { crates, third } \\
\text { mark, containing } 1 \text { arroba of candles (bee wax) } \\
(11,5 \mathrm{~kg}) \text {, white cotton clothes, blankets from } \\
\text { China }\end{array}$ & Filipinas,214,N.1,F.138r \\
\hline & $\begin{array}{l}\text { Sergeant } \\
\text { Major Agustín } \\
\text { Hernández }\end{array}$ & $\begin{array}{l}40 \text { crates, fourth mark, containing } 6 \text { arrobas of } \\
\text { candles (bee wax) }(69 \mathrm{~kg})\end{array}$ & Filipinas,214,N.1,F.139r \\
\hline
\end{tabular}




\begin{tabular}{|c|c|c|c|}
\hline $\begin{array}{l}\text { SHIPPER'S } \\
\text { MARK }\end{array}$ & SHIPPER & MERCHANDISE & SOURCE \\
\hline & $\begin{array}{l}\text { General Juan } \\
\text { de Echeverría }\end{array}$ & white cotton clothes, blankets from China & Filipinas,214,N.1,F.139v \\
\hline & $\begin{array}{l}\text { Captain } \\
\text { Fernando de } \\
\text { Angulo }\end{array}$ & $\begin{array}{l}\text { white cotton clothes, blankets from China, silks, } \\
\text { tapestries, towels, incense, cinnamon }\end{array}$ & Filipinas,214,N.1,F.141r \\
\hline & $\begin{array}{l}\text { Captain } \\
\text { Fernando de } \\
\text { Angulo }\end{array}$ & $\begin{array}{l}\text { cotton clothes from Cambay, blankets from } \\
\text { China, silks, damasks, towels, incense, clove }\end{array}$ & Filipinas,214,N.1,F.142r \\
\hline \multirow[t]{2}{*}{ No mark } & $\begin{array}{l}\text { Captain } \\
\text { Fernando de } \\
\text { Angulo }\end{array}$ & silks, fans, blankets & Filipinas,214,N.1,F.142v \\
\hline & $\begin{array}{c}\text { Captain } \\
\text { Antonio } \\
\text { Fernández de } \\
\text { Guevara }\end{array}$ & $\begin{array}{l}\text { white cotton clothes, blankets from China, silks, } \\
\text { damasks, towels, screens, writing desks }\end{array}$ & Filipinas,214,N.1,F.143r \\
\hline & $\begin{array}{c}\text { Captain } \\
\text { Atanasio } \\
\text { Fernández de } \\
\text { Guevara }\end{array}$ & $\begin{array}{c}\text { petticoats, blankets from China, white cotton } \\
\text { clothes }\end{array}$ & Filipinas,214,N.1,F.144r \\
\hline & $\begin{array}{c}\text { Sergeant Major } \\
\text { don Joseph de } \\
\text { Aguirre }\end{array}$ & cups, faience horses, blankets from llocos & Filipinas,214,N.1,F.144V \\
\hline & $\begin{array}{l}\text { Sergeant Major } \\
\text { don Joseph de } \\
\text { Aguirre }\end{array}$ & cups & Filipinas,214,N.1,F.145r \\
\hline
\end{tabular}




\begin{tabular}{|c|c|c|c|}
\hline $\begin{array}{l}\text { SHIPPER'S } \\
\text { MARK }\end{array}$ & SHIPPER & MERCHANDISE & SOURCE \\
\hline & $\begin{array}{l}\text { Captain } \\
\text { Bernardo de } \\
\text { Estrada }\end{array}$ & white cotton clothes, blankets from China & Filipinas,214,N.1,F.145r \\
\hline & $\begin{array}{c}\text { Second } \\
\text { Liutenant Pedro } \\
\text { Zapata }\end{array}$ & incense, cinnamon, pepper, faience & Filipinas,214,N.1,F.145V \\
\hline & $\begin{array}{l}\text { Captain } \\
\text { Francisco de } \\
\text { Ezpeleta }\end{array}$ & $\begin{array}{c}\text { cotton clothes from Cambay, blankets from } \\
\text { China }\end{array}$ & Filipinas,214,N.1,F.146v \\
\hline & $\begin{array}{l}\text { Captain } \\
\text { Francisco de } \\
\text { Ezpeleta }\end{array}$ & $\begin{array}{c}\text { cotton clothes from Cambay, blankets from } \\
\text { China, cotton clothes, damasks, screens, little } \\
\text { boxes }\end{array}$ & Filipinas,214,N.1,F.146v \\
\hline & $\begin{array}{c}\text { Captain } \\
\text { Antonio Ruiz } \\
\text { Moreno }\end{array}$ & $\begin{array}{c}\text { fabrics, blankets from China, white cotton } \\
\text { clothes, blankets }\end{array}$ & Filipinas,214,N.1,F.147v \\
\hline & $\begin{array}{l}\text { Captain Juan } \\
\text { de Arrese }\end{array}$ & $\begin{array}{l}\text { silks, satins, towels, blankets from China, trunks, } \\
\text { writing desks }\end{array}$ & Filipinas,214,N.1,F.149r \\
\hline & $\begin{array}{l}\text { Captain Luis } \\
\text { Antonio de } \\
\text { Tagle }\end{array}$ & $\begin{array}{l}\text { white cotton clothes, blankets from China, silks, } \\
\text { fans }\end{array}$ & Filipinas,214,N.1,F.150r \\
\hline & $\begin{array}{l}\text { Captain Luis } \\
\text { Antonio de } \\
\text { Tagle }\end{array}$ & white cotton clothes, blankets & Filipinas,214,N.1,F.150v \\
\hline
\end{tabular}




\begin{tabular}{|c|c|c|c|}
\hline SHIPPER'S & SHIPPER & MERCHANDISE & SOURCE \\
\hline & $\begin{array}{c}\text { Captain Luis } \\
\text { Antonio de } \\
\text { Tagle }\end{array}$ & white cotton clothes, blankets & Filipinas,214,N.1,F.151r \\
\hline & $\begin{array}{l}\text { Captain } \\
\text { Fernando de } \\
\text { Lemos }\end{array}$ & screens, satins, blankets, bowls & Filipinas,214,N.1,F.151r \\
\hline & $\begin{array}{l}\text { Captain Tomás } \\
\text { de Cruzeleygui }\end{array}$ & $\begin{array}{l}\text { white cotton clothes, blankets from China, } \\
\text { fabrics, combs }\end{array}$ & Filipinas,214,N.1,F.152v \\
\hline & $\begin{array}{l}\text { General Juan } \\
\text { de Echeverría }\end{array}$ & combs, bowls, blankets & Filipinas,214,N.1,F.153r \\
\hline & $\begin{array}{l}\text { General Juan } \\
\text { de Echeverría }\end{array}$ & towels, writing desk, fans & Filipinas,214,N.1,F.153v \\
\hline & $\begin{array}{l}\text { Sergeant Major } \\
\text { Joseph de } \\
\text { Aguirre }\end{array}$ & white cotton clothes, blankets & Filipinas,214,N.1,F.154r \\
\hline & $\begin{array}{l}\text { Sergeant Major } \\
\text { Joseph de } \\
\text { Aguirre }\end{array}$ & silks, fans, blankets & Filipinas,214,N.1,F.154v \\
\hline & $\begin{array}{l}\text { Sergeant Major } \\
\text { Joseph de } \\
\text { Aguirre }\end{array}$ & white cotton clothes, blankets & Filipinas,214,N.1,F.154v \\
\hline
\end{tabular}

